\title{
MAY 201965
}

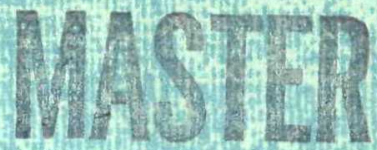

WANL-TME-574

OCTOBER, 1963

\section{NUCLEAR DATA LIBRARY FOR THE}

\section{FISSION PRODUCT INVENTORY PROGRAM}

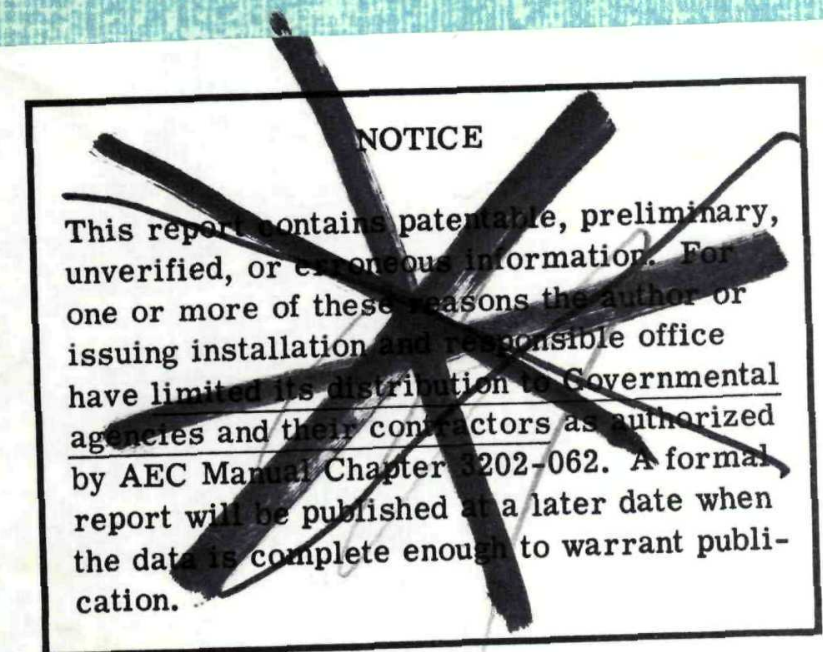

\section{NOT FOR PUBLIC PELETSE OFFLIAC

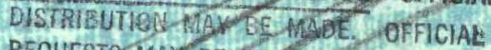 REQUESTS MAX BE-THEED REPORT CQNDANS WOTHINE OP PATENT INTEREST. PRCCZOURES ON EILE IN RECEIVINA SECTIONy $/ 2$ ?}

DISTRIBUTION OF THIS DOCUMENT UNLIMITED

\section{Astronuclear Laboratory Westinghouse Electric Corporation \\ RECEMED}




\section{DISCLAIMER}

This report was prepared as an account of work sponsored by an agency of the United States Government. Neither the United States Government nor any agency Thereof, nor any of their employees, makes any warranty, express or implied, or assumes any legal liability or responsibility for the accuracy, completeness, or usefulness of any information, apparatus, product, or process disclosed, or represents that its use would not infringe privately owned rights. Reference herein to any specific commercial product, process, or service by trade name, trademark, manufacturer, or otherwise does not necessarily constitute or imply its endorsement, recommendation, or favoring by the United States Government or any agency thereof. The views and opinions of authors expressed herein do not necessarily state or reflect those of the United States Government or any agency thereof. 


\section{DISCLAIMER}

Portions of this document may be illegible in electronic image products. Images are produced from the best available original document. 


$$
\text { NOTICE }
$$

WANL-TME-574

NoTiCE October, 1963 This report was prepared as an Government. Neither This United States Goved States Energy sponsored States nor the United the United Stevelopment Administration, contractors, Research and Developmer any of their contractors, their employees, nor any employees, makes any subcontractors, or thir lied, or assumes any leness warranty, express or impled, accuracy, completeness liability or responsibilinformation, apparatus, product not or usefulness of any information, that its use would not process disclosed, or represents

process disclosely owned rights.

\section{NUCLEAR DATA LIBRARY FOR THE FISSION PRODUCT PROGRAM}

\section{By: M. R. Trammell}

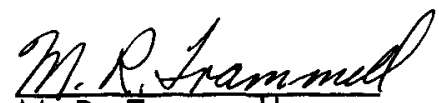

W. A Jemninger

Safeguards Engineering Dept.

\section{APPROVED:}

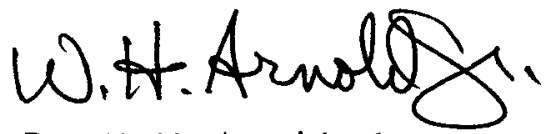

Dr. W. H. Arnold, Jr.

Deputy Manager, Analysis
INFORMATION CATEGORY

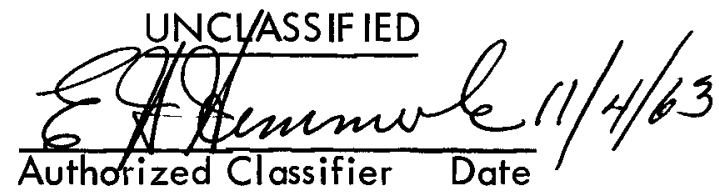




\section{NUCLEAR DATA LIBRARY FOR THE FISSION PRODUCT PROGRAM}

I. Table of Nuclear Data

The Table of Nuclear Data includes the atomic number and chemical symbol, mass number, half-life, decay constant, fission yield, $\beta$-spectrum, and $\gamma$-spectrum of each nucl ide.

1. Half-lives and the decay constants computed from them are compatible with those used by P. Zigman's group at the USNRDL ${ }^{(9)}$. Abbreviations used in the list of half-lives are:

$$
\begin{aligned}
& \text { S - Seconds } \\
& M \text { - Minutes } \\
& \mathrm{H} \text { - Hours } \\
& \text { D - Days } \\
& \text { Y - Years }
\end{aligned}
$$

Decay constants are given in reciprocal seconds and, e.g., $.72963-2=$ $.72963 \times 10^{-2}$.

2. Fission yields given are yields from thermal neutron fission of $U^{235}$ and have been taken from Weaver, Strom, and Killeen ${ }^{(8)}$. In those cases where no energy spectra are available for a particular nuclide, and the nuclide does not contribute significantly to the total activity $(<0.1 \%$ based on the FPIP), the fission yield for that nuclide has been assigned 
to the next succeeding chain member. This re-assignment has been carried out only for early chain members and in no case has an entire chain been deleted.

3. $\beta$-and $\gamma$-spectral data have been obtained from the sources indicated in the Table. Abbreviations refer to:

$\begin{array}{cc}\text { NDS } & \text { reference } \\ \text { NUS } & \text { reference } \\ \text { SHS } & \text { reference } \\ \text { Stehn } & \text { reference }\end{array}$

Those references which appear with an asterisk (NDS* and NUS*) indicate that the details of the energy spectrum are not known but that the $Q$-value for the disintegration of that nuclide is available in the source given. In these cases, it has been assumed that half the

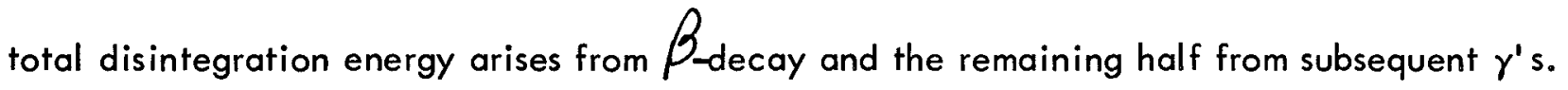
The total decay has been divided accordingly in order to arrive at a more real istic estimate of the total fission energy spectrum than would be possible otherwise.

All decay energies are given in Mev.

\section{Table of Decay Chains}

The Table of Decay Chains is based on the table compiled by W. Henninger ${ }^{(2)}$ after consultation with the Applied Research Branch, U. S. Naval Radiological Defense Laboratory. The fission yield for the initial member of each chain has been obtained as described above and a few modifications of the original compilation have been made on 
the basis of the decay schemes given in Nuclear Data Sheets. Where two or more isomers of the same nuclide appear, the fission yield given will be split equally between them. In those cases where isomers became initial chain members, the branching ratio for the decay of the parent nuclide has been taken into account.

The total chain yield is given at the end of each chain. 


\section{BIBLIOGRAPHY}

1. Bolles, R.C., and Ballou, N. E., "Calculated Activities and Abundances of U 235 Fission Products, " U.S. Naval Radiological Defense Laboratory No. 456 (1956).

2. Henninger, W. A., Private communcations.

3. Katcoff, S. K., "Fission Product Yields from Neutron Induced Fission, " Nucleonics, $18,201(1960)$.

4. "Nuclear Data Sheets, "National Academy of Sciences - National Research Council, Washington, D.C.

5. Nuclear Utility Services, Inc., "Environmental Safety Aspects of Nuclear Rocket Flight Operations, "NUS-144 (July, 1963).

6. Stehn, John F., "Table of Radioactive Nuclides." Nucleonics, 18, 186 (1960).

7. Strominger, D., Hollander, J.M., and Seaborg, G.T., "Table of Isotopes, "Rev. Mod. Physics, 30, 2 Part II, (1958).

8. Weaver, L. E., Strom, P.O., and Killeen, P.A., "Estimated Total Chain and Independent Fission Yields for Several Neutron-Induced Fission Processes, " USNRDL-TR-633, (March, 1963).

9. Zigman, P., Private communication. 


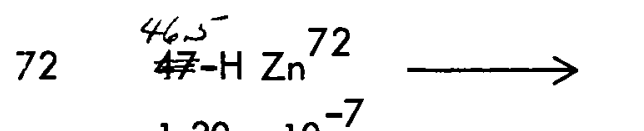

$14.1-\mathrm{H} \mathrm{Ga}^{72}$

$\longrightarrow \quad$ Stable Ge $\mathrm{T}^{72}$

$1.30 \times 10^{-7}$

$1.00 \times 10^{-8}$

0

$1.4 \times 10^{-7}$

73

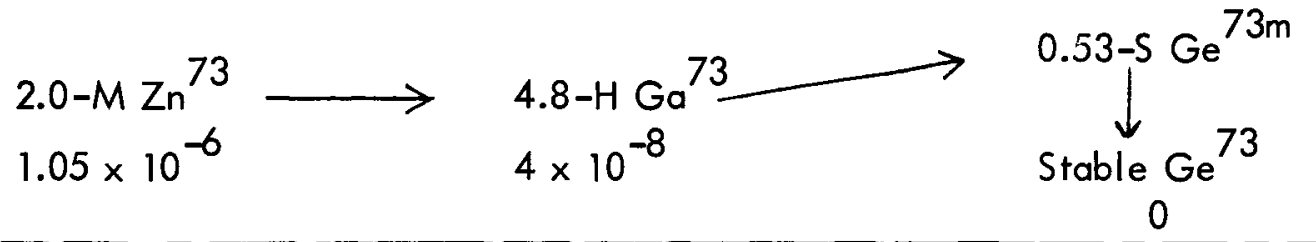

Stable $G e^{74}$

$\begin{array}{rr}74 & 7.8-\mathrm{M} \mathrm{Ga}^{74} \\ 3.44 \times 10^{-6} & \longrightarrow\end{array} \quad \begin{array}{r}\text { Stable } \\ \hline\end{array}$

0

$3.44 \times 10^{-6}$

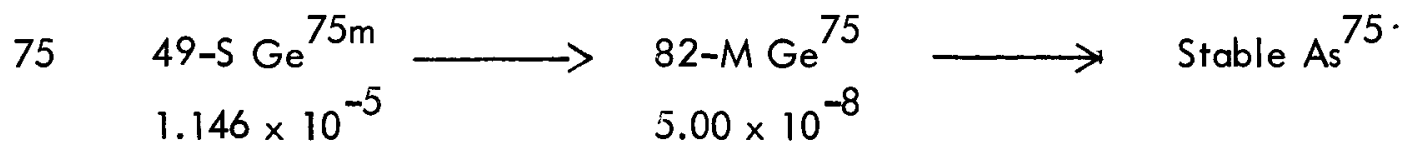

$1.151 \times 10^{-5}$

$76 \quad 30-\mathrm{S} \mathrm{Ga}^{76} \longrightarrow \quad$ Stable $\mathrm{Ge}^{76}$

$3.322 \times 10^{-5} \quad 1.81 \times 10^{-6}$

$3.503 \times 10^{-5}$ 
Chain Yield

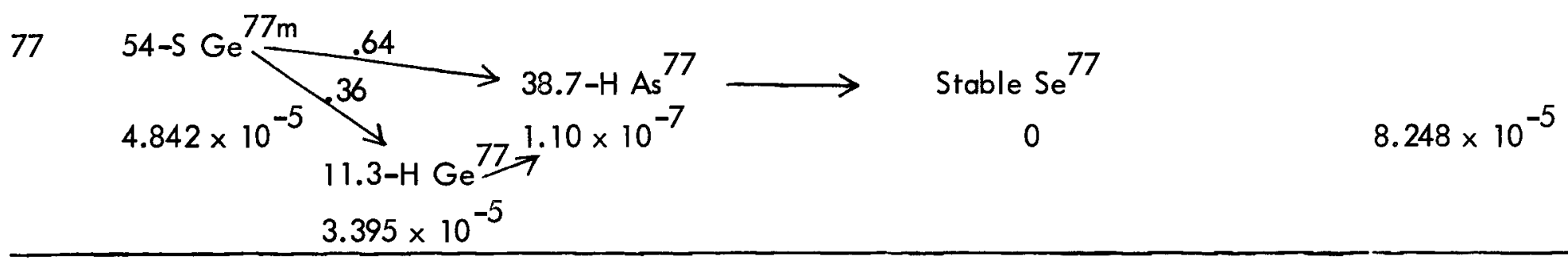

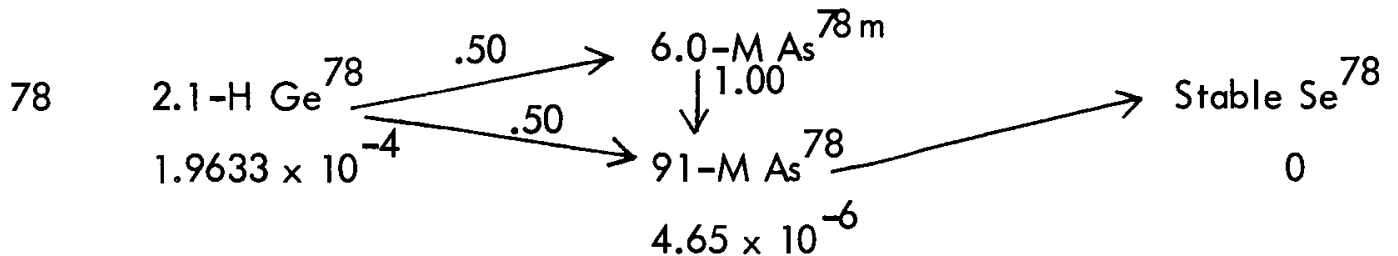

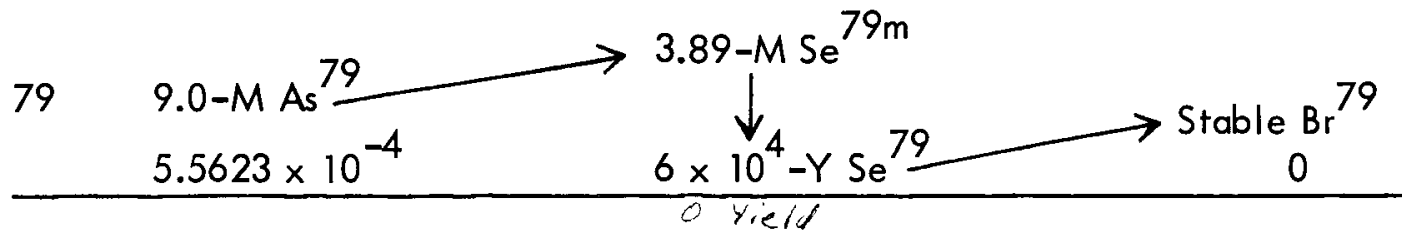

$5.5622 \times 10^{-4}$

$80 \quad 36-\mathrm{SAs}^{80} \longrightarrow$ Stable Se ${ }^{80}$

$9.3827 \times 10^{-4} \quad 3.28 \times 10^{-6} \quad 9.4156 \times 10^{-4}$

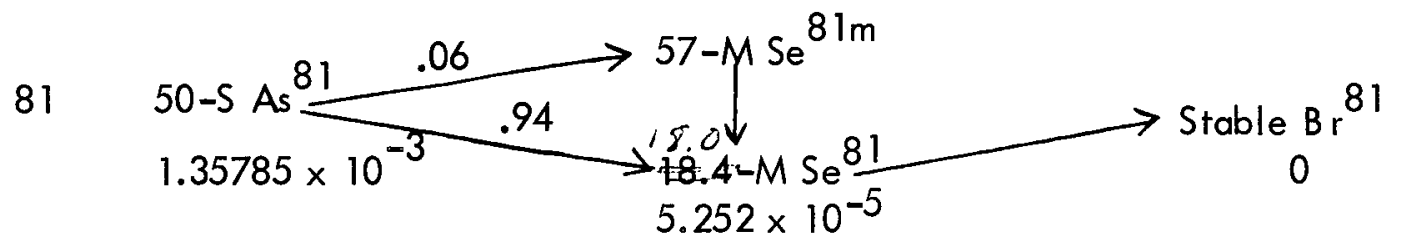


Sheet No. 3

Chain Yield

\begin{tabular}{lll}
\hline 82 & $18-\mathrm{S} \mathrm{As}^{82} \longrightarrow$ & Stable Se \\
$2.28093 \times 10^{-3}$ & $1.3906 \times 10^{-4}$ & $2.41998 \times 10^{-3}$ \\
\hline
\end{tabular}

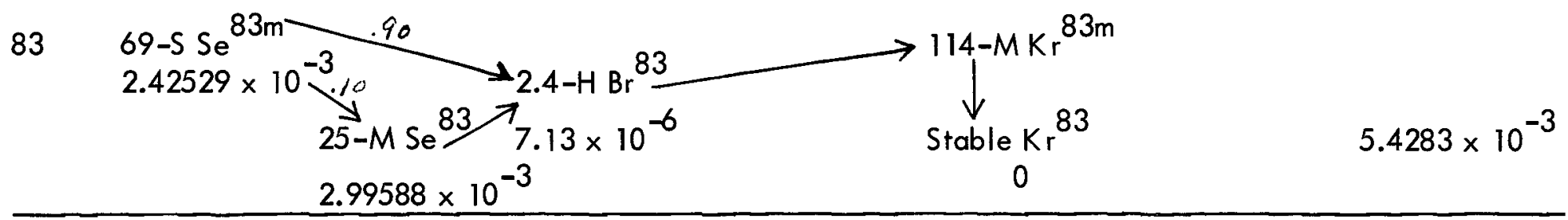

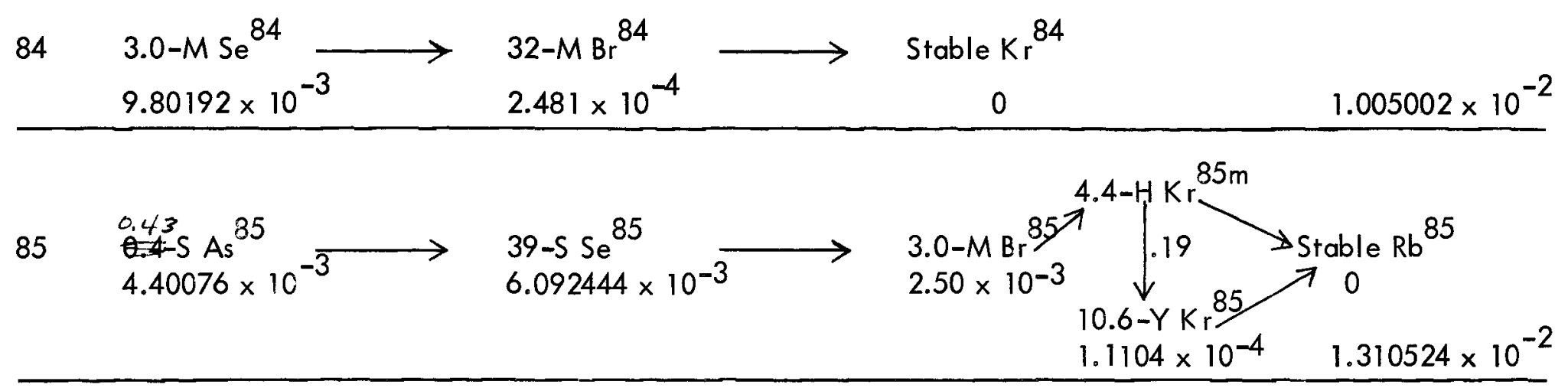

$86 \quad 16-\mathrm{S} \mathrm{Se}^{86} \longrightarrow 60-\mathrm{SBr}^{86} \longrightarrow$ Stable Kr $^{86}$

$1.250019 \times 10^{-2} \quad 6.4926 \times 10^{-3} \quad 1.11044 \times 10^{-3} \quad 2.010324 \times 10^{-3}$

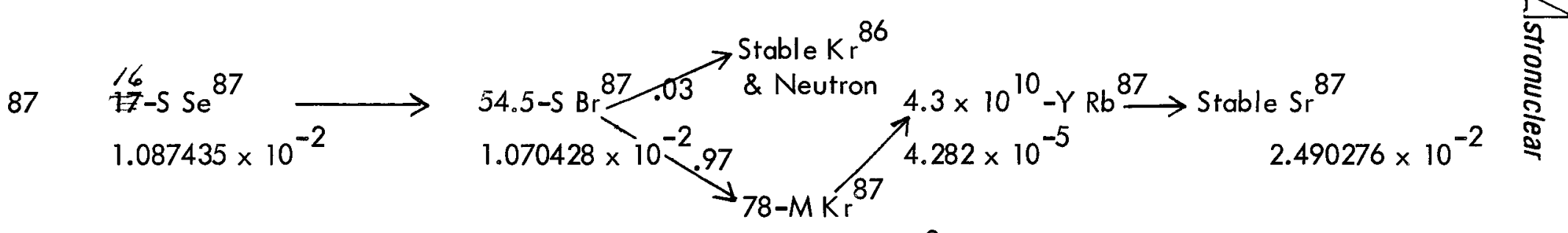

$$
3.28131 \times 10^{-3}
$$




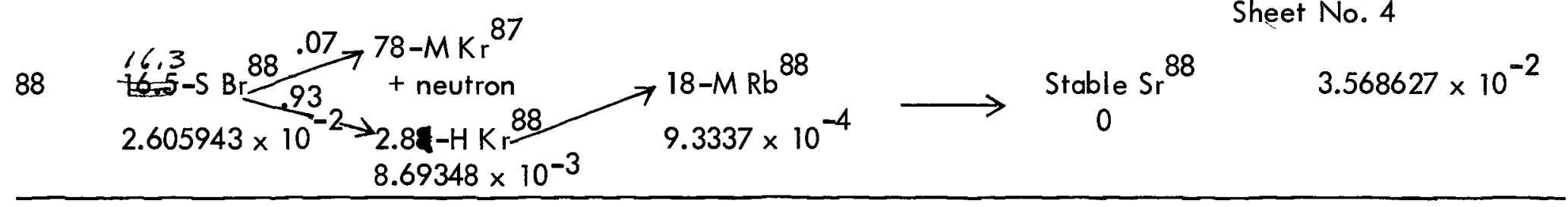

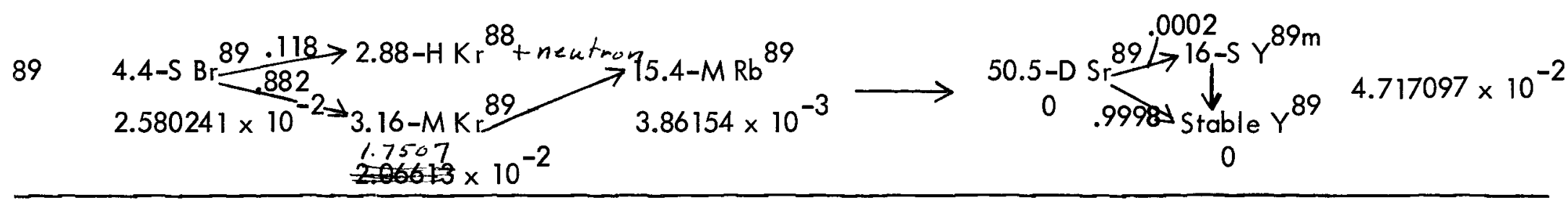

$90 \quad 33-\mathrm{S} \mathrm{Kr} P 0$
$4.418467 \times 10^{-2}$

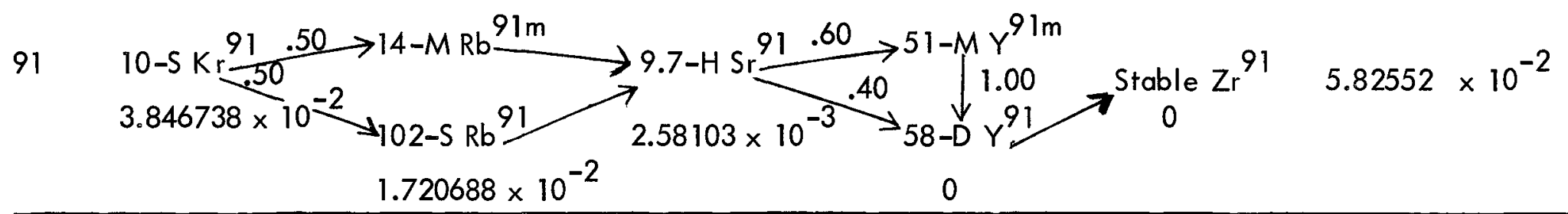

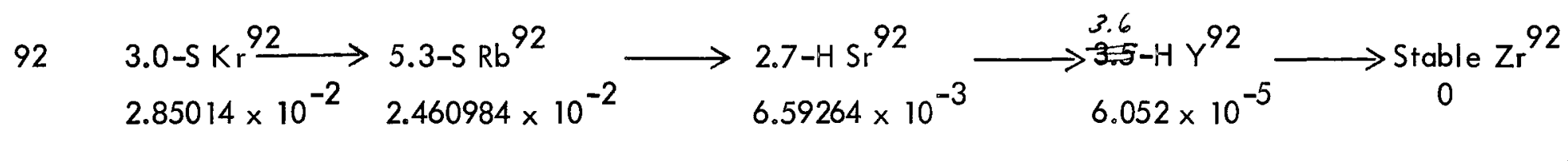
$5.97644 \times 10^{-2}$ 
Sheet No. 5

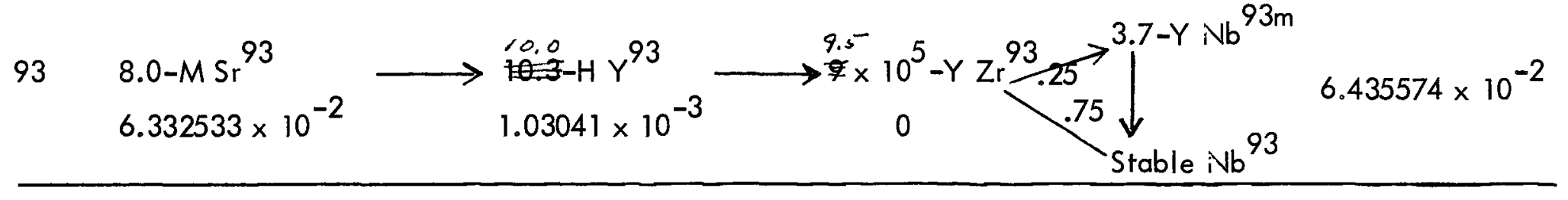

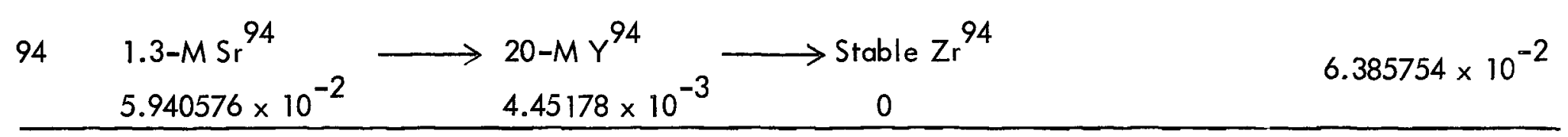

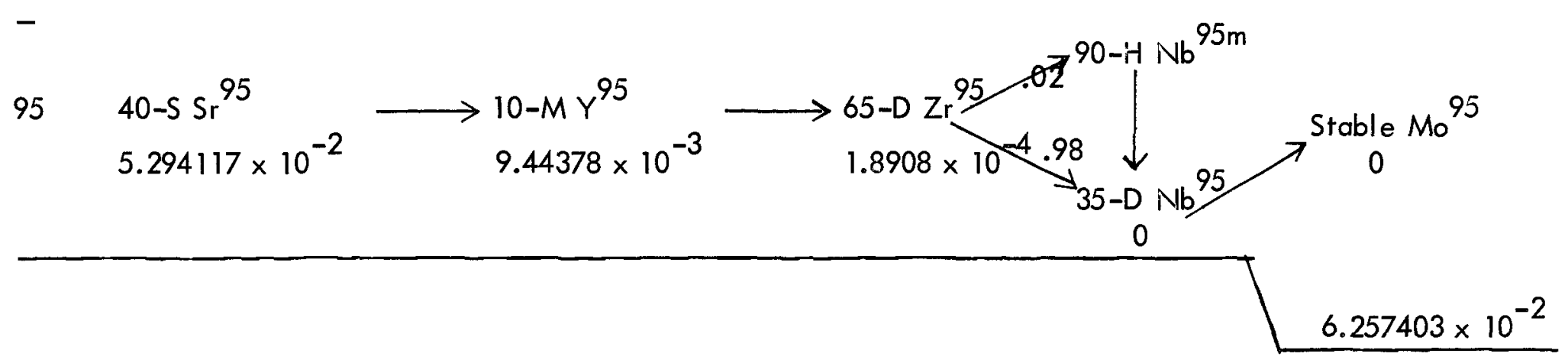

$96 \quad 2.3-\mathrm{M} \mathrm{Y}^{96} \longrightarrow$ Stable $\mathrm{Zr}^{96}$

$6.02821 \times 10^{-2} \quad 2.27091 \times 10^{-3} \quad 6.255302 \times 10^{-2}$

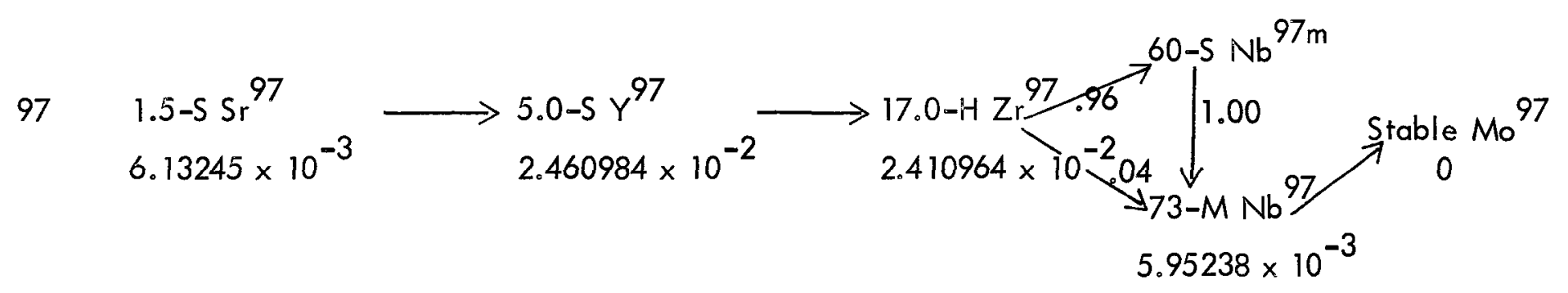




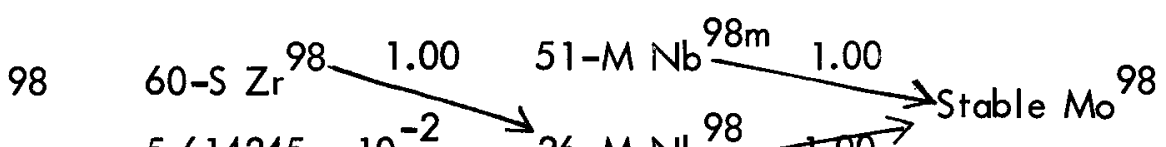

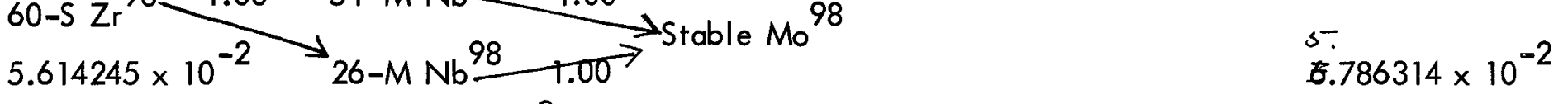

$1.72069 \times 10^{-3}$

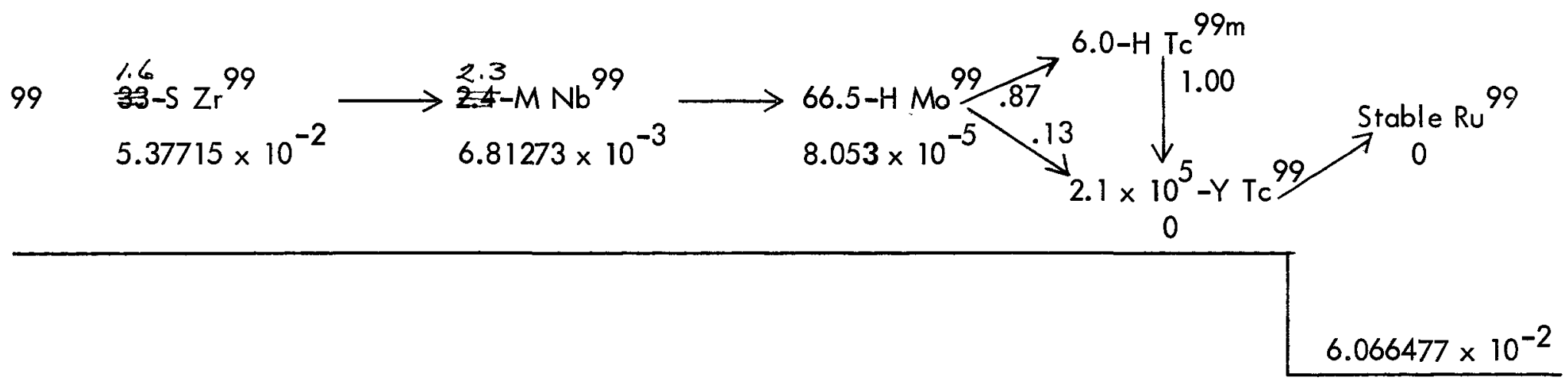

\begin{tabular}{ll}
$100 \quad \begin{array}{l}3.0-\mathrm{M} \mathrm{Nb}^{100} \\
6.021908 \times 10^{-2}\end{array}$ & $\begin{array}{l}\text { Stable Mo }{ }^{100} \\
2.80112 \times 10^{-3}\end{array}$ \\
\hline
\end{tabular}

$\begin{array}{ll}101 & 1.0-\mathrm{M} \mathrm{Nb}^{101} \\ 4.2507 \times 10^{-2}\end{array} \longrightarrow \begin{gathered}14.6-\mathrm{M} \mathrm{Mo}^{101} \\ 7.60304 \times 10^{-3}\end{gathered} \longrightarrow \begin{gathered}14.0-\mathrm{M} \mathrm{Tc}^{101} \\ 1.5706 \times 10^{-4}\end{gathered} \longrightarrow$ Stable Ru $^{101}$

102

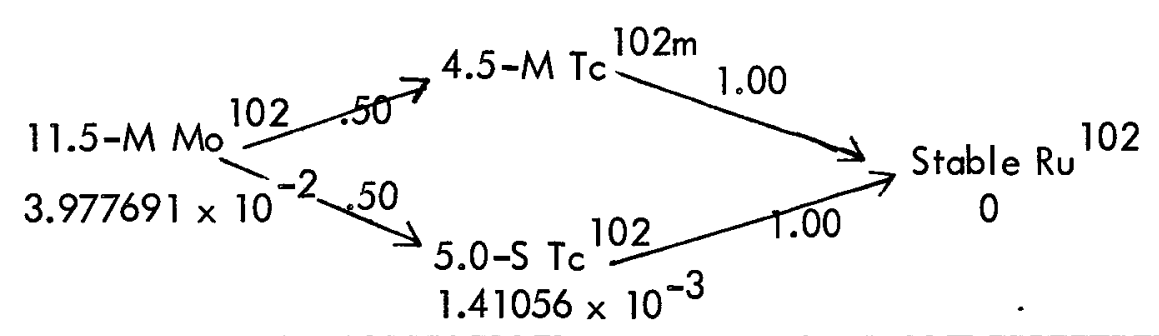




$$
103 \quad 72-\mathrm{S} \mathrm{Tc}^{103} \longrightarrow 39.7-\mathrm{D} \mathrm{Ru} \stackrel{0}{2.978291 \times 10^{-2}} \underbrace{103}_{\substack{.005 \\ \text { Stable Rh } \\ 0}}
$$

\begin{tabular}{|c|c|c|c|c|}
\hline \multirow[t]{2}{*}{104} & $2.5-M_{M o}{ }^{104}$ & $18-M T_{c}^{104}$ & Stable Ru ${ }^{104}$ & \\
\hline & $1.446278 \times 10^{-2}$ & $3.40136 \times 10^{-3}$ & $1.4006 \times 10^{-4}$ & $1.30042 \times 10^{-2}$ \\
\hline
\end{tabular}
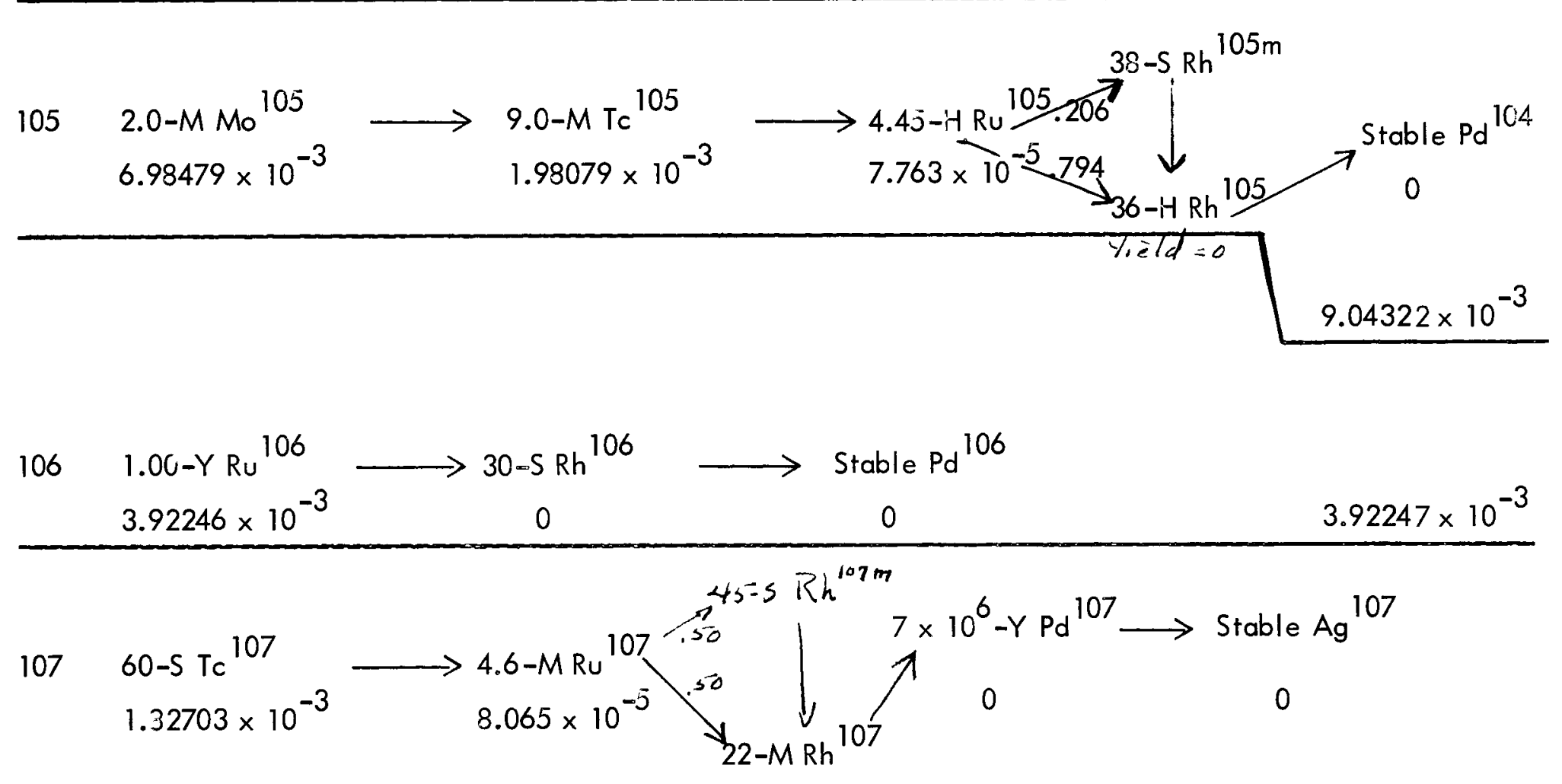

0 


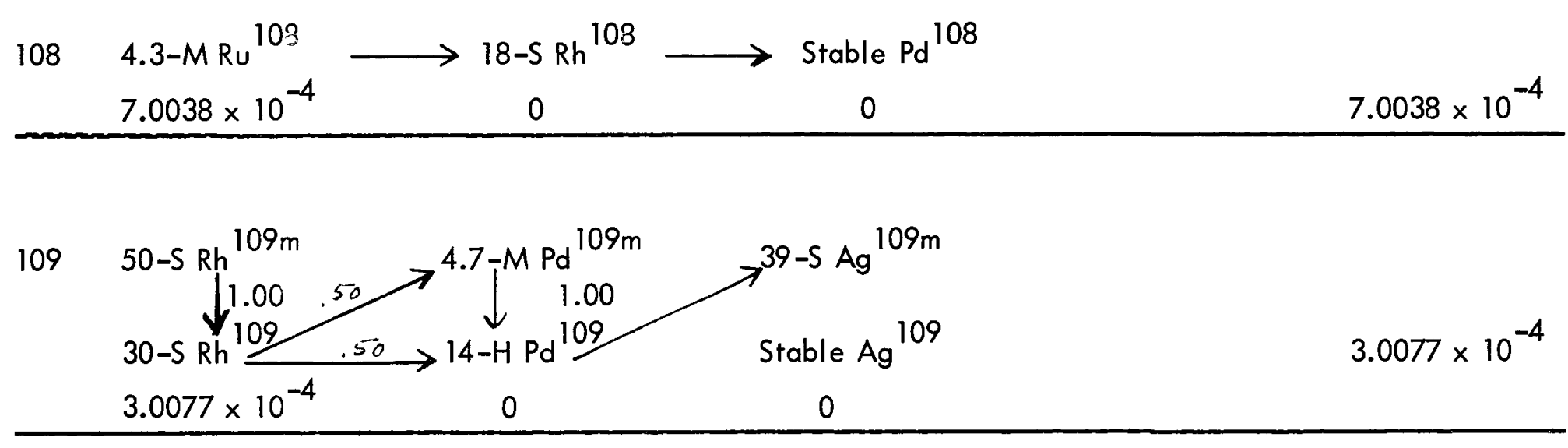

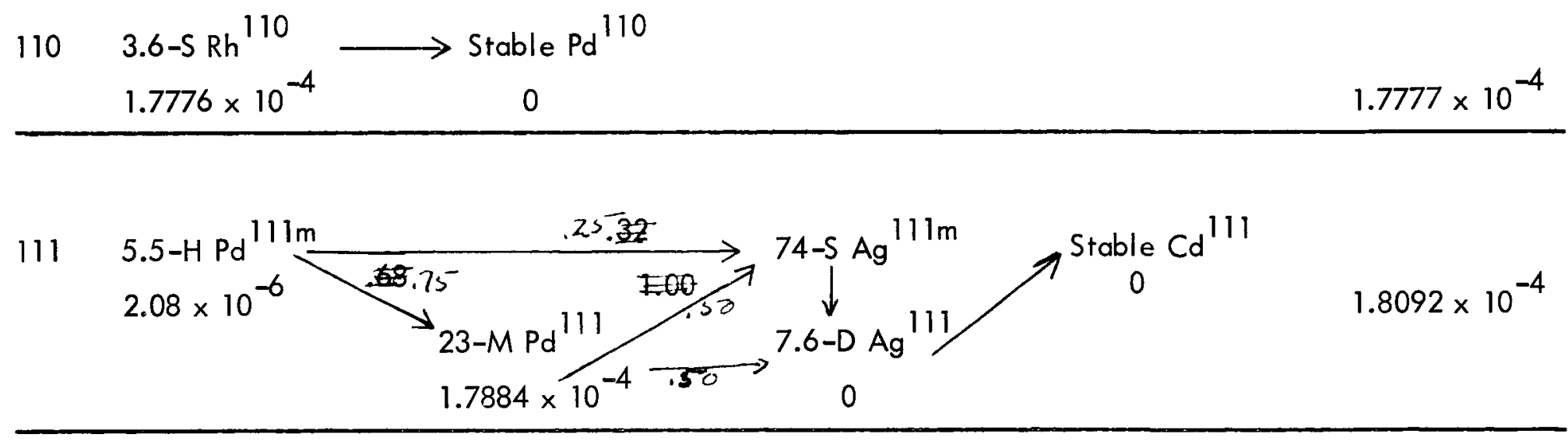

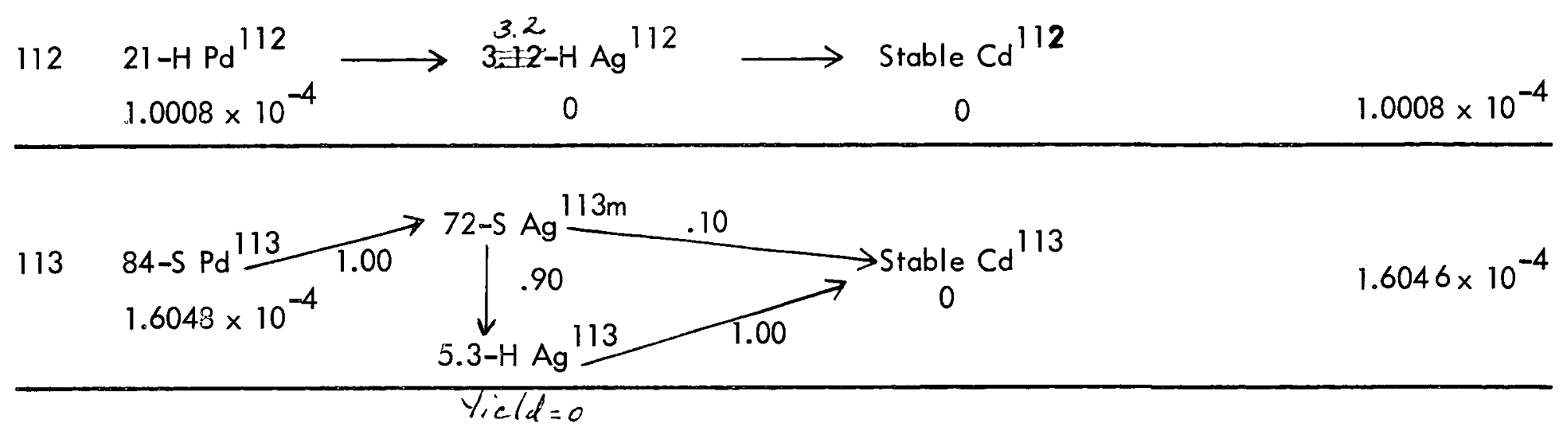




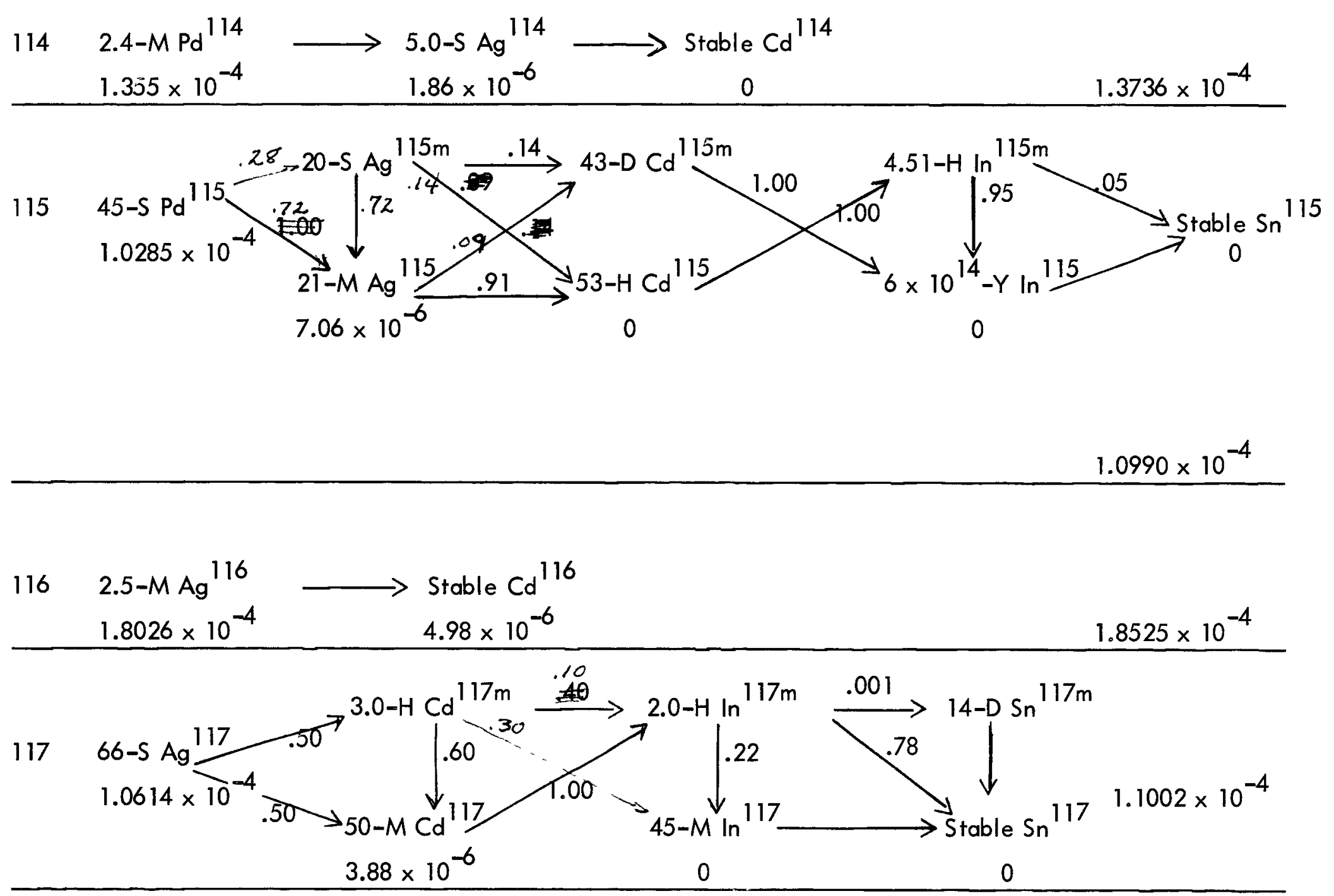

\begin{tabular}{llccc}
118 & $50-\mathrm{M} \mathrm{Cd}^{118}$ \\
$1.4236 \times 10^{-4}$ & 0 & $5.0-\mathrm{S} \mathrm{In}^{118}$ & $\longrightarrow$ & Stable Sn $^{118}$ \\
& 0 & 0 & $1.4236 \times 10^{-4}$ \\
\hline
\end{tabular}


129

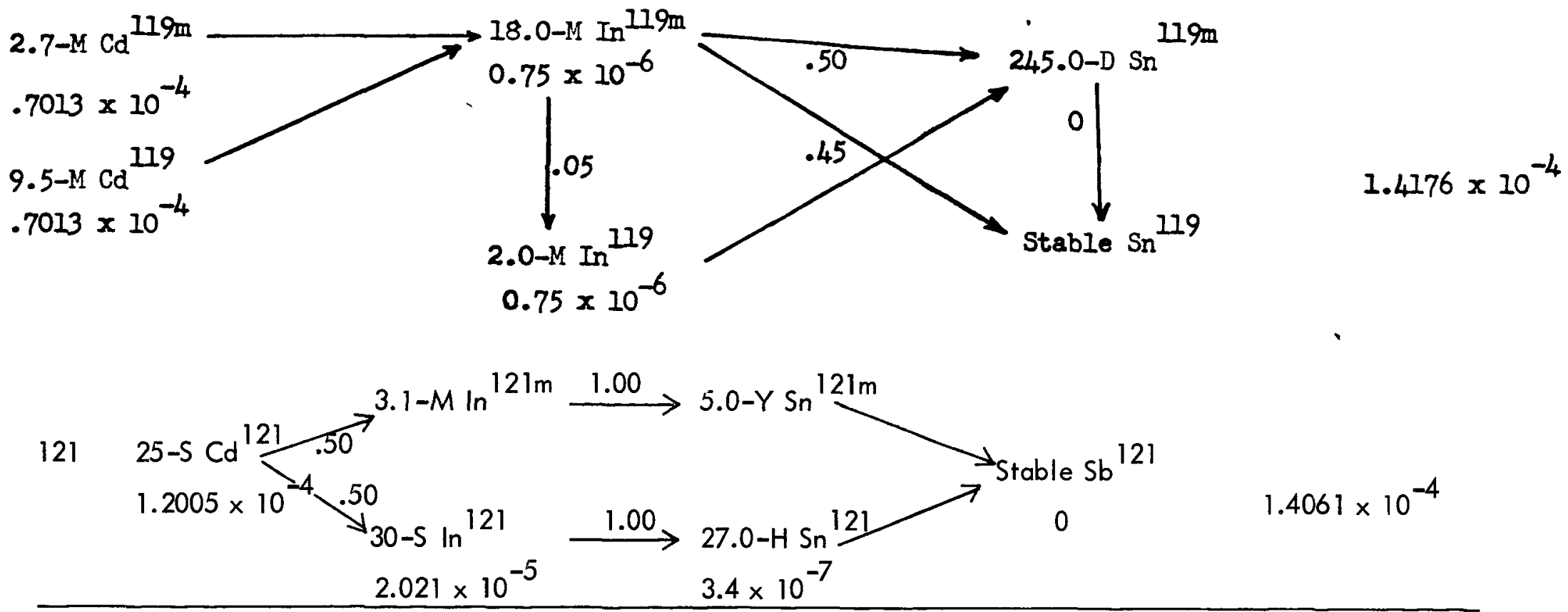

$12245-\mathrm{S} \mathrm{In}^{122}$
$1.4366 \times 10^{-4}$ $\begin{aligned} & \text { Stable Sn }^{122} \\ & 7.09 \times 10^{-6}\end{aligned}$ $1.5075 \times 10^{-4}$

$123 \quad 36-S \ln ^{123 m} 1.00 \quad 125-D^{123}{ }^{123 m} 1.00$

$10-5 \ln \stackrel{123 \quad 1.00}{\longrightarrow} 441-M_{\text {Sn }} \stackrel{123 \quad 1.00 \longrightarrow}{\longrightarrow}$ Stable Sb ${ }^{123}$

$1.6146 \times 10^{-4}$ $1.4627 \times 10^{-4} \quad 1.521 \times 10^{-5}$

$12420-5 \ln ^{124} \longrightarrow$ Stable Sn ${ }^{124}$

$1.4175 \times 10^{-4} \quad 3.461 \times 10^{-5}$

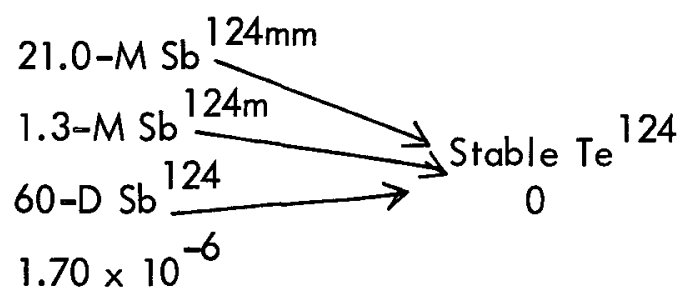




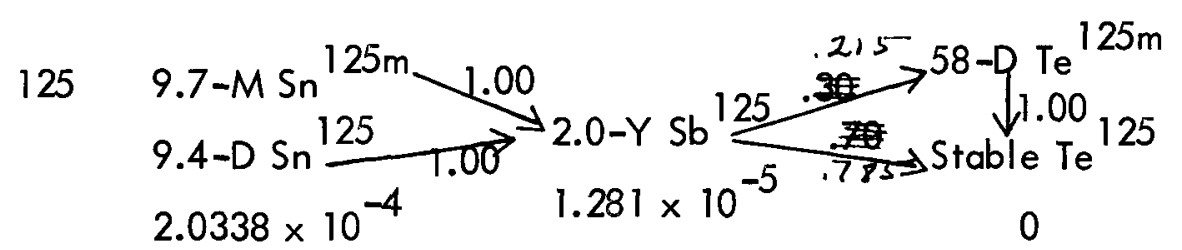

$1262 \times 10^{5}-\mathrm{Sn}^{126}+.00^{19-M \mathrm{Sb}^{126 \mathrm{~m}}} \cdot 99 \mathrm{SStable} \mathrm{Te}^{126}$

$2.7472 \times 10^{-4} \quad 12.5-\mathrm{D} \mathrm{Sb}{ }^{126} 1.00^{7} 6.7 \times 10^{-7}$

$4.392 \times 10^{-5}$
$2.1619 \times 10^{-4}$

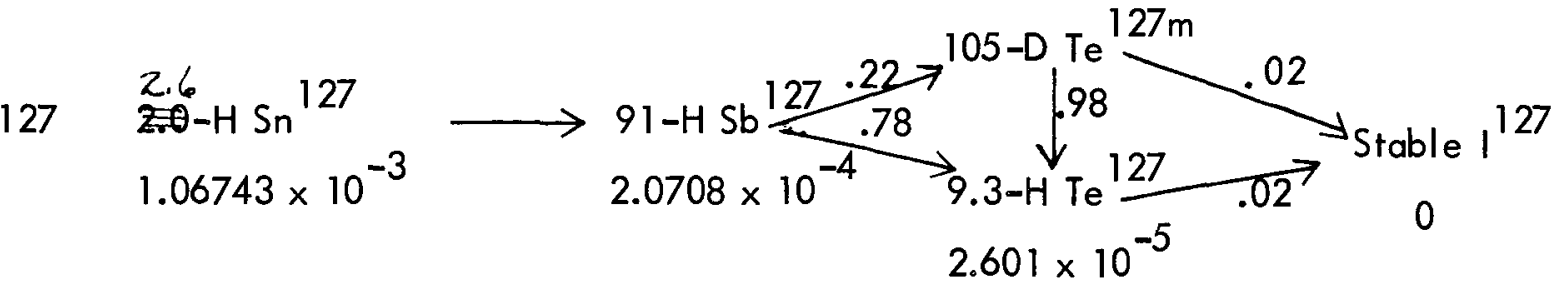

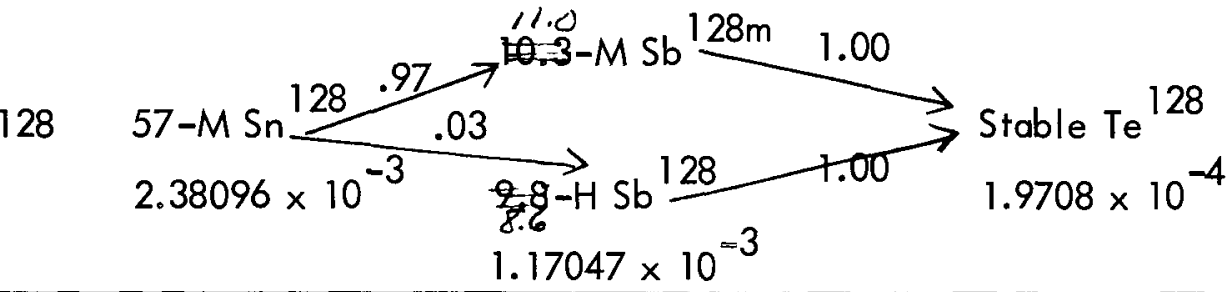

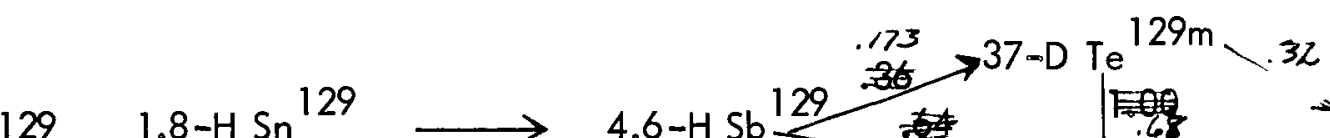

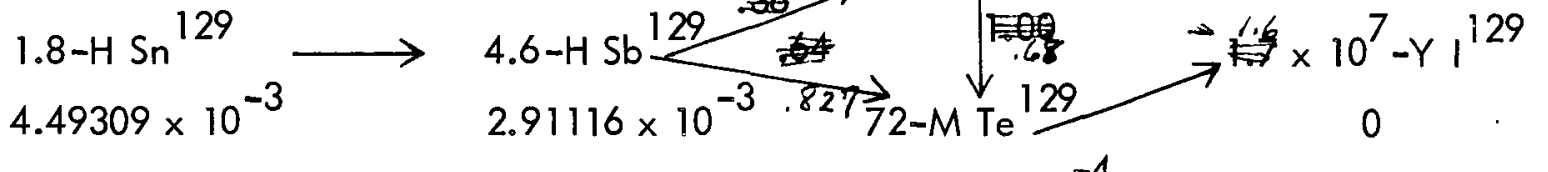
$6.1925 \times 10^{-4}$ 
Sheet No. 13

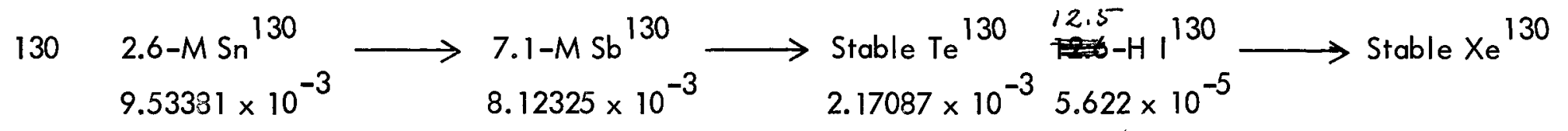

$1.988415 \times 10^{-2}$

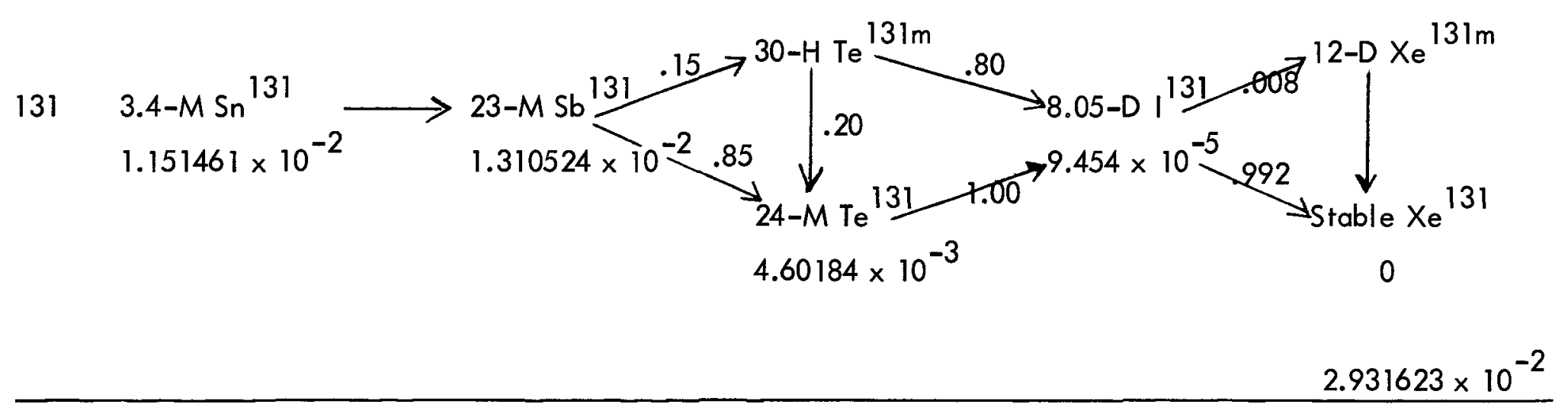

$132 \underset{2.2-\mathrm{M} \mathrm{Sn}^{132}}{9.31972 \times 10^{-3}} \longrightarrow \begin{aligned} & 2.1-\mathrm{M} \mathrm{Sb}^{132} \\ & 2.060824 \times 10^{-2}\end{aligned} \longrightarrow \begin{aligned} & 78-\mathrm{H} \mathrm{Te}^{132} \\ & 1.220488 \times 10^{-2}\end{aligned} \longrightarrow \begin{aligned} & 2.3-\mathrm{H} \mathrm{I}^{132} \\ & 1.67067 \times 10^{-3}\end{aligned} \longrightarrow$

Stable Xe $\quad 4.380352 \times 10^{-2}$ 0

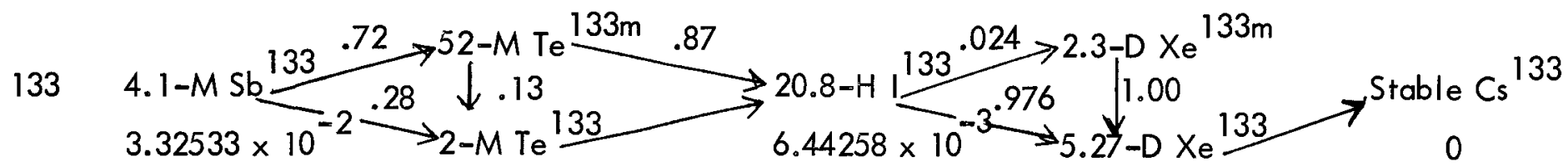
$2.611044 \times 10^{-2}$ 0 
134

$48-\mathrm{S} \mathrm{Sb}^{134}$

$1.805722 \times 10^{-2}$
${ }^{42}-\mathrm{M} \mathrm{Te}^{134}$

$3.781513 \times 10^{-2}$ $\longrightarrow 53-\mathrm{M} \mathrm{I}^{134}$

$2.170868 \times 10^{-2}$ $\longrightarrow$ Stable $\mathrm{Xe}^{134}$

$2.75110 \times 10^{-3}$

Chain Yield: $\quad 8.033213 \times 10^{-2}$

$$
\begin{aligned}
& 135 \quad 6.0-\mathrm{S} \mathrm{Sb}^{135} \longrightarrow 1.4-\mathrm{M} \mathrm{Te}^{135} \\
& 4.37175 \times 10^{-3} \quad 2.250900 \times 10^{-2}
\end{aligned}
$$

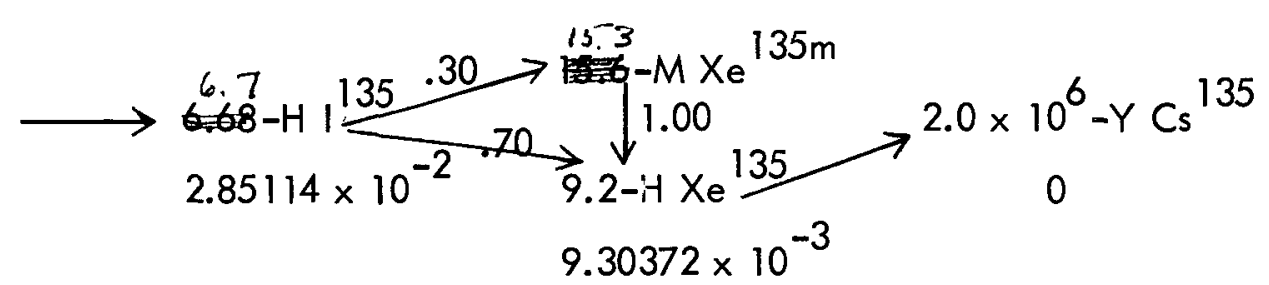

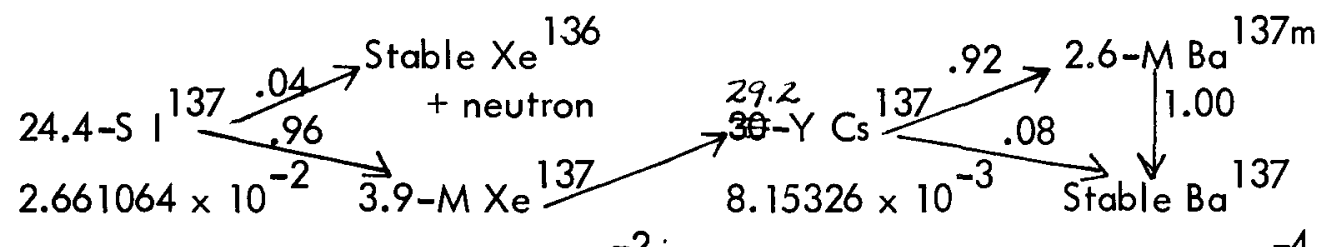

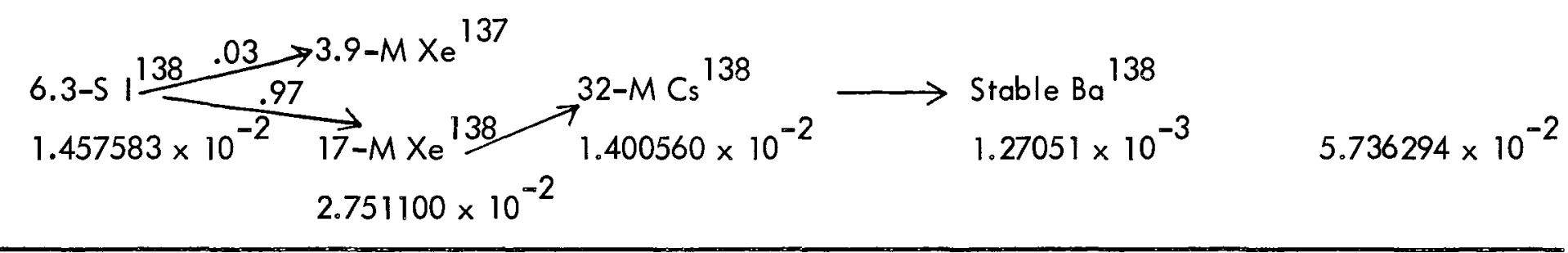


139

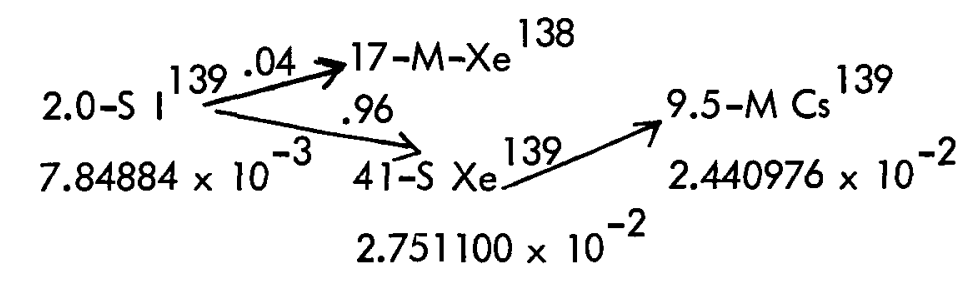

Sheet No. 15

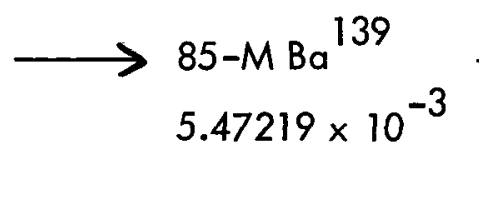

Chain Yield: $\quad 6.524180 \times 10^{-2}$

\begin{tabular}{|c|c|c|c|c|}
\hline \multirow[t]{3}{*}{140} & $\begin{array}{l}16-5 \times e^{140} \\
2.306922 \times 10^{-2}\end{array}$ & $\begin{array}{l}66-S C_{s}^{140} \\
2.961184 \times 10^{-2}\end{array}$ & $\begin{array}{l}12.8-\mathrm{D} \mathrm{Ba}^{140} \\
1.120448 \times 10^{-2}\end{array}$ & $\begin{array}{l}40.2-\mathrm{H} \mathrm{La}^{140} \\
3.7115 \times 10^{-4}\end{array} \longrightarrow$ \\
\hline & & & Stable $C e^{140}$ & \\
\hline & & & 0 & $6.425670 \times 10^{-2}$ \\
\hline
\end{tabular}

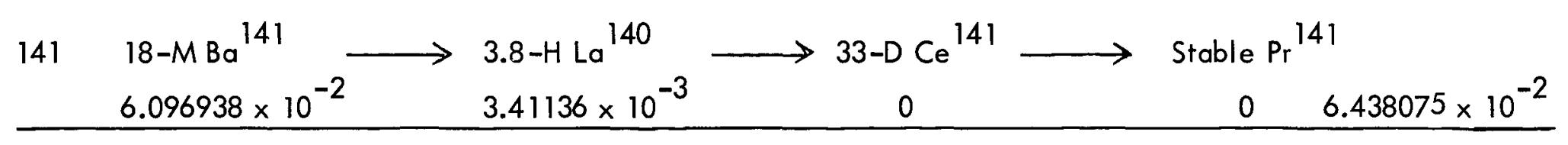

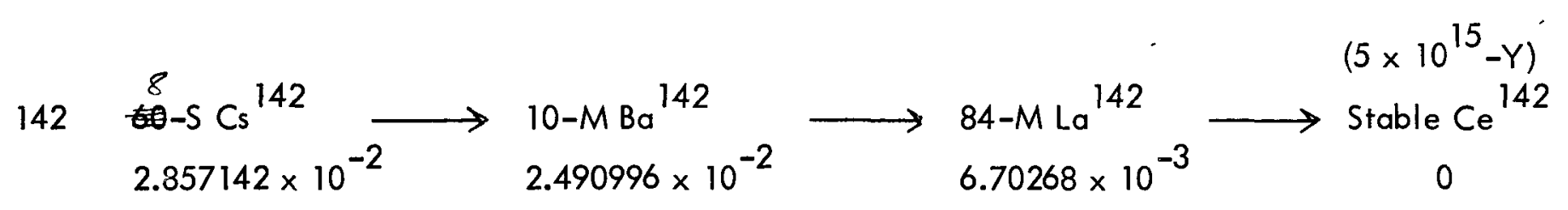

Chain Yield: $\quad 6.018407 \times 10^{-2}$

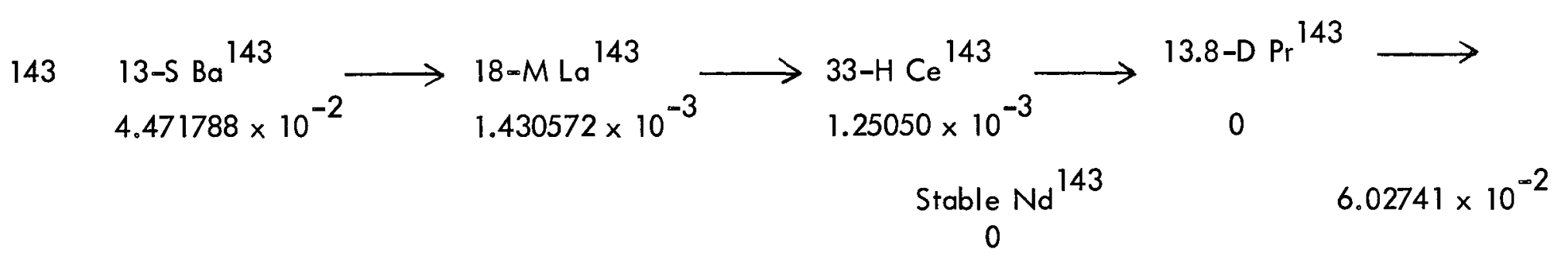


Sheet No. 16

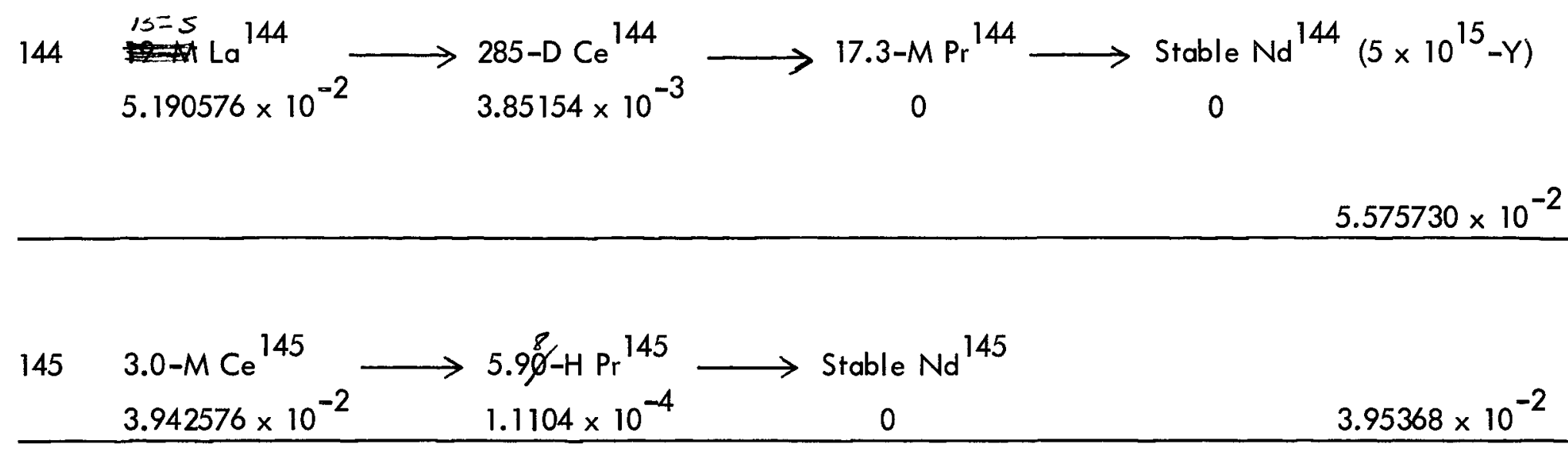

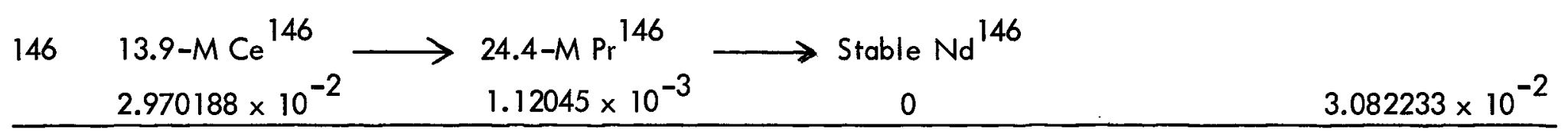

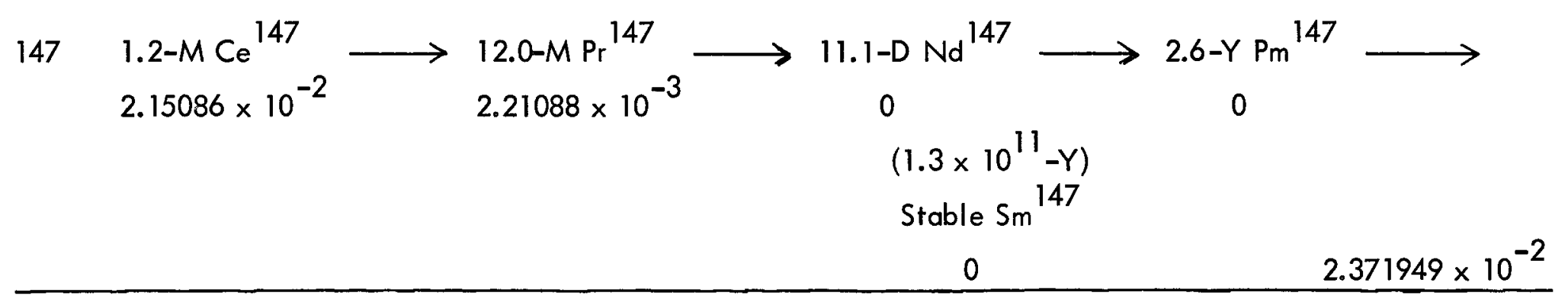

$148 \quad 1.95-\mathrm{MPr}^{148} \longrightarrow$ Stable Nd $^{148}$

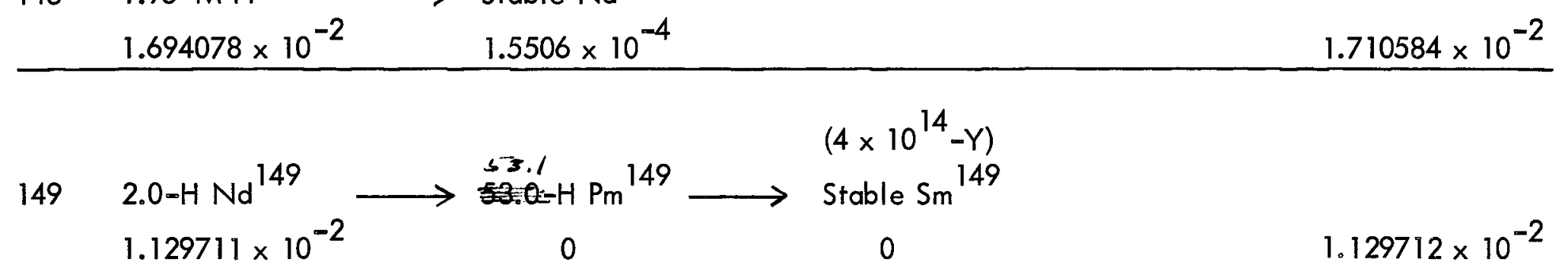


$150 \quad 15-\mathrm{S} \mathrm{Pr}^{150} \longrightarrow$ Stable Nd ${ }^{150} 2.7-\mathrm{H} \mathrm{Pm}^{150} \longrightarrow$ Stable Sm $^{150}$

$4.45528 \times 10^{-3} \quad 1.70068 \times 10^{-3} \quad 2.1208 \times 10^{-4} \quad 0 \quad 6.36805 \times 10^{-3}$

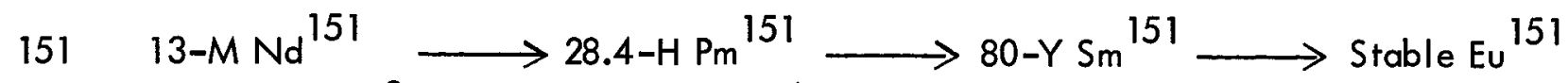

$4.19327 \times 10^{-3} \quad 2.3209 \times 10^{-4} \quad 0 \quad 0 \quad 0 \quad 4.42537 \times 10^{-3}$

\begin{tabular}{llll}
152 & $3.0-\mathrm{M} \mathrm{Nd}^{152}$ \\
$2.39696 \times 10^{-3}$ & $6.0-\mathrm{M} \mathrm{Pm}^{152}$ & Stable Sm & \\
& $3.8315 \times 10^{-4}$ & $5.92 \times 10^{-6}$ & $2.78603 \times 10^{-3}$ \\
\hline
\end{tabular}

\begin{tabular}{|c|c|c|c|c|}
\hline 153 & ${ }^{3.5} \mathrm{Mm}^{153}$ & $47-\mathrm{S} \mathrm{Sm}^{153}$ & Stable Eu ${ }^{153}$ & \\
\hline & $1.65206 \times 10^{-3}$ & $6.032 \times 10^{-5}$ & 0 & $1.71238 \times 10^{-3}$ \\
\hline
\end{tabular}

\begin{tabular}{lll}
154 & $\begin{array}{l}2.5 \\
\end{array}$ \\
$6.9167 \times 10^{-4}$ & Stable Sm ${ }^{154}$ \\
& $7.163 \times 10^{-5}$ & $7.633 \times 10^{-4}$ \\
\hline
\end{tabular}

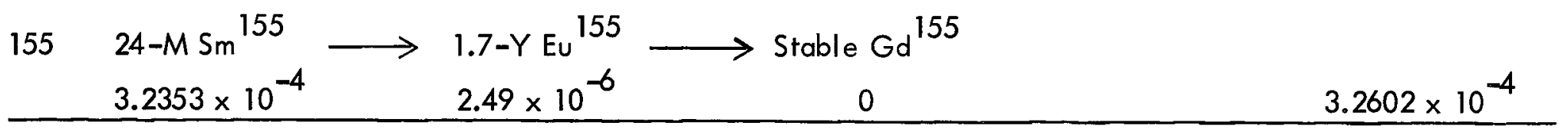

$156 \begin{gathered}10-\mathrm{H} \mathrm{Sm}^{156} \\ 1.3358 \times 10^{-4}\end{gathered} \longrightarrow \begin{gathered}15.4-\mathrm{D} \mathrm{Eu}^{156} \\ 7.38 \times 10^{-6}\end{gathered} \longrightarrow$ Stable Gd $^{156}$ 
$157 \quad 15.0-\mathrm{H} \mathrm{Eu}^{157} \longrightarrow$ Stable Gd $^{157}$
$7.719 \times 10^{-5}$
$9 \times 10^{-8}$
Decay Chain: $7.729 \times 10^{-5}$

$158 \quad 60-\mathrm{M} \mathrm{Eu}^{158} \longrightarrow$ Stable Gd ${ }^{158}$

$1.975 \times 10^{-5} \quad 3.9 \times 10^{-7}$

$2.015 \times 10^{-5}$

$159 \quad 18.0-\mathrm{H} \mathrm{Gd}^{159} \longrightarrow{\text { Stable } \mathrm{Tb}^{159}}^{159}$

$1.047 \times 10^{-5}$

0

$1.047 \times 10^{-5}$

$16070-\mathrm{S} \mathrm{Eu}^{160} \longrightarrow$ Stable Gd $^{160} \quad \mathrm{73- \textrm {D } \mathrm { Tb }}{ }^{160} \longrightarrow$ Stable Dy $^{160}$

$2.80 \times 10^{-6} \quad 4.9 \times 10^{-7} \quad 2 \times 10^{-8} \quad 3.31 \times 10^{-6}$

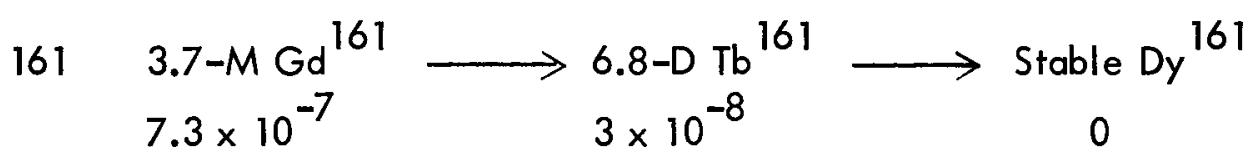

0

$7.6 \times 10^{-7}$ 


\begin{tabular}{|c|c|c|c|c|c|c|c|c|c|c|c|c|}
\hline \multirow{2}{*}{$\frac{\text { Nuclide }}{30 \mathrm{Zn}}$} & \multirow{2}{*}{$\begin{array}{r}\begin{array}{r}\text { Mass } \\
\text { No. }\end{array} \\
72\end{array}$} & \multicolumn{2}{|l|}{ Half-life } & \multicolumn{2}{|c|}{$\begin{array}{c}\text { Decay Constant } \\
\left(\mathrm{sec}^{-1}\right)\end{array}$} & \multicolumn{2}{|c|}{$\begin{array}{l}\text { Fission Yield } \\
\text { (Fractional) }\end{array}$} & \multirow{2}{*}{$\begin{array}{c}\mathrm{E}_{\boldsymbol{\beta}}(\max ) \\
0.3 \\
1.6\end{array}$} & \multicolumn{3}{|c|}{ Energy of Radiation } & \multirow{2}{*}{$\begin{array}{l}\text { Reference } \\
\text { NDS }\end{array}$} \\
\hline & & 47 & $H$ & .40966 & -5 & 1300 & -6 & & $\begin{array}{r}95 \\
5\end{array}$ & & & \\
\hline & 73 & 2.0 & M & .57762 & -2 & 1050 & -5 & 2.5 & 100 & 2.5 & 100 & NDS* \\
\hline \multirow[t]{3}{*}{$31 \mathrm{Ga}$} & 72 & 14.1 & $\mathrm{H}$ & .13655 & -4 & .1000 & -7 & $\begin{array}{l}0.66 \\
0.68 \\
0.959 \\
1.508 \\
1.94 \\
2.529 \\
3.166\end{array}$ & $\begin{array}{r}17 \\
24 \\
35 \\
7 \\
7 \\
5 \\
5\end{array}$ & $\begin{array}{l}0.601 \\
0.630 \\
0.786 \\
0.812 \\
0.835 \\
0.894 \\
1.050 \\
1.231 \\
1.268 \\
1.465 \\
1.598 \\
1.68 \\
1.860 \\
2.201 \\
2.490 \\
2.508\end{array}$ & $\begin{array}{c}7.2 \\
4.5 \\
3 \\
2.6 \\
69 \\
8 \\
5.3 \\
1.2 \\
1.4 \\
23.5 \\
10.3 \\
1.5 \\
5.3 \\
29 \\
9 \\
17\end{array}$ & NDS \\
\hline & 73 & 4.8 & $\mathrm{H}$ & .40113 & -4 & .4000 & -7 & $\begin{array}{r}\sim 0.40 \\
1.19 \\
1.48\end{array}$ & $\begin{array}{r}7 \\
91 \\
\leq 2\end{array}$ & $\begin{array}{l}0.295 \\
0.745 \\
1.04\end{array}$ & $\begin{array}{r}97 \\
6 \\
1\end{array}$ & NDS \\
\hline & 74 & 7.8 & M & .14811 & -2 & .3440 & -5 & $\begin{array}{l}1.1 \\
1.84 \\
2.05 \\
2.27 \\
2.65 \\
4.3\end{array}$ & $\begin{array}{r}11 \\
4 \\
3 \\
10 \\
69 \\
3\end{array}$ & $\begin{array}{l}0.49 \\
0.596 \\
0.60 \\
0.87 \\
1.00 \\
1.10 \\
1.20 \\
1.31\end{array}$ & $\begin{array}{r}7.5 \\
76 \\
3.7 \\
9.5 \\
7.4 \\
6 \\
11 \\
2.2\end{array}$ & NDS \\
\hline
\end{tabular}




\begin{tabular}{|c|c|c|c|c|c|c|c|c|c|c|c|c|}
\hline \multirow[t]{2}{*}{ Nuclide } & \multirow[t]{2}{*}{$\begin{array}{c}\text { Mass } \\
\text { No. }\end{array}$} & \multicolumn{2}{|c|}{ Half-life } & \multicolumn{2}{|c|}{$\begin{array}{l}\text { Decay Constant } \\
\left(\sec ^{-1}\right)\end{array}$} & \multicolumn{2}{|c|}{$\begin{array}{l}\text { Fission Yield } \\
\text { (Fractional) }\end{array}$} & \multicolumn{4}{|c|}{ Energy of Radiation } & \multirow[t]{2}{*}{ Reference } \\
\hline & & & & & & & & & & $\begin{array}{l}1.45 \\
1.60 \\
1.72 \\
1.84 \\
1.96 \\
2.20 \\
2.35 \\
2.55 \\
2.72 \\
2.95 \\
3.17 \\
3.33 \\
3.6\end{array}$ & $\begin{array}{r}8 \\
0.4 \\
6.4 \\
2.8 \\
5.5 \\
0.9 \\
58.8 \\
2.5 \\
4 \\
3.1 \\
1.8 \\
1.8 \\
1.3\end{array}$ & \\
\hline \multirow{2}{*}{$32 \mathrm{Ge}$} & $75 \mathrm{~m}$ & 49 & $\mathrm{~S}$ & .14146 & -1 & .1146 & -4 & 0 & 0 & 0.139 & 100 & NDS \\
\hline & 75 & 82 & $M$ & .14088 & -3 & .5000 & -7 & $\begin{array}{r}0.55 \\
\sim 0.72 \\
0.92 \\
0.98 \\
1.19\end{array}$ & $\begin{array}{c}0.41 \\
0.29 \\
11.4 \\
1.4 \\
86.5\end{array}$ & $\begin{array}{l}0.066 \\
0.199 \\
0.265 \\
0.427 \\
0.477\end{array}$ & $\begin{array}{c}0.3 \\
1.4 \\
11 \\
0.3 \\
0.3\end{array}$ & NDS \\
\hline $32 \mathrm{Ge}$ & $77 \mathrm{~m}$ & 54 & $S$ & .12836 & -1 & .4842 & -4 & $\begin{array}{l}2.7 \\
2.9\end{array}$ & $\begin{array}{l}18 \\
46\end{array}$ & $\begin{array}{l}0.159 \\
0.215\end{array}$ & $\begin{array}{l}36 \\
18\end{array}$ & NUS \\
\hline
\end{tabular}




\begin{tabular}{|c|c|c|c|c|c|c|c|c|c|c|c|c|}
\hline \multirow[b]{2}{*}{ Nuclide } & \multirow{2}{*}{$\begin{array}{c}\begin{array}{c}\text { Mass } \\
\text { No. }\end{array} \\
77\end{array}$} & \multirow{2}{*}{\multicolumn{2}{|c|}{ Half-Life }} & \multirow{2}{*}{\multicolumn{2}{|c|}{$\begin{array}{l}\text { Decay Constant } \\
\qquad\left(\mathrm{sec}^{-1}\right)\end{array}$}} & \multirow{2}{*}{\multicolumn{2}{|c|}{$\begin{array}{r}\text { Fission Yield } \\
\text { (Fractional) }\end{array}$}} & \multicolumn{4}{|c|}{ Energy of Radiation } & \multirow{2}{*}{$\begin{array}{c}\text { Reference } \\
\text { NUS }\end{array}$} \\
\hline & & & & & & & & $E_{\beta}(\max )$. & $\%$ & & $\%$ & \\
\hline & 78 & 2.1 & $H$ & .91687 & -4 & .19633 & -3 & 0.9 & 100 & 0 & 0 & NDS \\
\hline \multirow[t]{2}{*}{33 As } & 77 & 38.7 & $\mathrm{H}$ & .49752 & -5 & .1100 & -6 & $\begin{array}{l}0.16 \\
0.438 \\
0.684\end{array}$ & $\begin{array}{r}2.8 \\
2.8 \\
94.4\end{array}$ & $\begin{array}{l}0.246 \\
0.524\end{array}$ & $\begin{array}{l}2.8 \\
2.7\end{array}$ & NDS \\
\hline & $78 m$ & 6.0 & $M$ & .19254 & -2 & .2325 & -5 & 0 & 0 & 0.50 & 100 & NDS \\
\hline
\end{tabular}




\begin{tabular}{|c|c|c|c|c|c|c|c|c|c|c|c|c|}
\hline Nuclide & $\begin{array}{l}\text { Mass } \\
\text { No. }\end{array}$ & \multicolumn{2}{|c|}{ Half-Life } & \multicolumn{2}{|c|}{$\begin{array}{l}\text { Decay Constant } \\
\left(\sec ^{-1}\right)\end{array}$} & \multicolumn{2}{|c|}{$\begin{array}{l}\text { Fission Yield } \\
\text { (Fractional) }\end{array}$} & \multicolumn{4}{|c|}{ Energy of Radiation } & $\begin{array}{c}-4- \\
\text { Reference }\end{array}$ \\
\hline & 79 & 9.0 & $M$ & .12836 & -2 & .55623 & -3 & 1.1 & 100 & 1.1 & 100 & NDS* \\
\hline & 80 & 36 & $\mathrm{~S}$ & .19254 & -1 & .93827 & -3 & 3.25 & 100 & 3.25 & 100 & NDS* \\
\hline & 85 & 0.4 & $S$ & .17329 & +1 & .440076 & -2 & 4 & 100 & 4 & 100 & NDS* \\
\hline \multirow[t]{3}{*}{$34 \mathrm{Se}$} & $79 m$ & 3.89 & $M$ & .29698 & -2 & 0 & & 0 & 0 & 0.096 & 100 & NUS \\
\hline & 79 & $6 \times 10^{4}$ & Y & .36608 & -12 & \multicolumn{2}{|l|}{ 。 } & 0.16 & 100 & 0 & 0 & Stehn \\
\hline & $81 \mathrm{~m}$ & 57 & $M$ & .20268 & -3 & .2626 & -4 & 0 & 0 & 0.103 & 100 & NUS \\
\hline \multirow[t]{3}{*}{8} & 81 & 18.4 & $M$ & .62785 & -3 & .2626 & -4 & 1.38 & 100 & 0 & 0 & NUS \\
\hline & $83 m$ & 69 & $\mathrm{~S}$ & .10046 & -1 & .242529 & -2 & $\begin{array}{l}1.5 \\
3.4\end{array}$ & $\begin{array}{l}10 \\
90\end{array}$ & $\begin{array}{l}0.35 \\
0.65 \\
1.01 \\
2.02\end{array}$ & $\begin{array}{l}<10 \\
<10 \\
5 \\
5\end{array}$ & NUS \\
\hline & 84 & 3.0 & $M$ & .38508 & -2 & .980192 & -2 & 0.95 & 100 & 0.95 & 100 & N'JS* \\
\hline
\end{tabular}




\begin{tabular}{|c|c|c|c|c|c|c|c|c|c|c|c|c|}
\hline \multirow{3}{*}{ Nuclide } & \multirow{3}{*}{$\begin{array}{l}\begin{array}{l}\text { Mass } \\
\text { No. }\end{array} \\
85\end{array}$} & \multirow{2}{*}{\multicolumn{2}{|c|}{ Half-life }} & \multirow{2}{*}{\multicolumn{2}{|c|}{$\begin{array}{l}\text { Decay Constant } \\
\quad\left(\sec ^{-1}\right)\end{array}$}} & \multirow{2}{*}{\multicolumn{2}{|c|}{$\begin{array}{l}\text { Fission Yield } \\
\text { (Fractional) }\end{array}$}} & \multicolumn{4}{|c|}{ Energy of Radiation } & \multirow[b]{2}{*}{ Reference } \\
\hline & & & & & & & & $E_{\beta}(\max )$ & $\%$ & & $\%$ & \\
\hline & & 39 & $S$ & .17773 & -1 & .609244 & -2 & 2.5 & 100 & 2.5 & 100 & NUS* \\
\hline & 86 & 16 & $\mathrm{~s}$ & .43322 & -1 & .1250019 & -1 & 2 & 100 & 2 & 100 & NDS* \\
\hline & 87 & 17 & $S$ & .40774 & -1 & 1087435 & -1 & 4 & 100 & 4 & 100 & NUS* \\
\hline \multirow[t]{24}{*}{$35 \mathrm{Br}$} & 83 & 2.4 & $\mathrm{H}$ & .80226 & -4 & .7130 & -5 & $\begin{array}{l}0.91 \\
0.96\end{array}$ & $\underset{80}{20}$ & 0.046 & 20 & NUS \\
\hline & 84 & 32 & $M$ & .36102 & -3 & 2481 & -3 & 0.50 & 3 & $\begin{array}{l}0.27 \\
0.35\end{array}$ & $\begin{array}{l}0.5 \\
1.0\end{array}$ & NDS \\
\hline & & & & & & & & 0.77 & 19 & 0.43 & 2.1 & \\
\hline & & & & & & & & 0.98 & 2 & 0.47 & 0.9 & \\
\hline & & & & & & & & 1.39 & 14 & 0.52 & 2.4 & \\
\hline & & & & & & & & 1.81 & 1.5 & 0.61 & 2.3 & \\
\hline & & & & & & & & 2.80 & 15 & 0.74 & 2.9 & \\
\hline & & & & & & & & 3.83 & 14 & 0.81 & 6.3 & \\
\hline & & & & & & & & 4.68 & 32 & 0.880 & 41.7 & \\
\hline & & & & & & & & & & 1.01 & 8.8 & \\
\hline & & & & & & & & & & 1.21 & 3.2 & \\
\hline & & & & & & & & & & 1.47 & 1.7 & \\
\hline & & & & & & & & & & 1.51 & 1.0 & \\
\hline & & & & & & & & & & 1.74 & 2.0 & \\
\hline & & & & & & & & & & 1.90 & 15.0 & \\
\hline & & & & & & & & & & 2.05 & 1.6 & \\
\hline & & & & & & & & & & 2.17 & 1.6 & \\
\hline & & & & & & & & & & 3.03 & 3.0 & \\
\hline & & & & & & & & & & 3.28 & 2.4 & \\
\hline & & & & & & & & & & 3.93 & 10.4 & \\
\hline & 85 & 3.0 & $M$ & .38508 & -2 & .2501 & -2 & 2.5 & 100 & 0 & 0 & NUS \\
\hline & 86 & 60 & $s$ & .11552 & -1 & .64926 & -2 & 4 & 100 & 4 & 100 & NDS* \\
\hline & 87 & 54.5 & $s$ & .12718 & -1 & 1070428 & -1 & $\begin{array}{l}2.6 \\
8.0\end{array}$ & $\begin{array}{l}70 \\
30\end{array}$ & $\sim \frac{3}{5.4}$ & $3 \frac{14}{36}$ & NUS \\
\hline & 88 & 16.5 & $S$ & .42009 & -1 & .2605943 & -1 & 4.85 & 100 & 4.85 & 100 & NDS* \\
\hline
\end{tabular}




\begin{tabular}{|c|c|c|c|c|c|c|c|c|c|c|c|c|}
\hline \multirow{3}{*}{ Nuclide } & \multirow{3}{*}{$\begin{array}{c}\begin{array}{c}\text { Mass } \\
\text { No. }\end{array} \\
89\end{array}$} & \multirow{2}{*}{\multicolumn{2}{|c|}{ Half-life }} & \multirow{2}{*}{\multicolumn{2}{|c|}{$\begin{array}{l}\text { Decay Constant } \\
\left(\sec ^{-1}\right)\end{array}$}} & \multirow{2}{*}{\multicolumn{2}{|c|}{$\begin{array}{l}\text { Fission Yield } \\
\text { (Fractional) }\end{array}$}} & \multicolumn{4}{|c|}{ Energy of Radiation } & \multirow{3}{*}{$\frac{\begin{array}{c}-6- \\
\text { Reference }\end{array}}{\text { NDS* }}$} \\
\hline & & & & & & & & $E_{\beta}(\max )$. & $\%$ & & $\%$ & \\
\hline & & .4 .4 & $s$ & .15753 & 0 & .2580241 & -1 & 4.5 & 100 & 4.5 & 100 & \\
\hline \multirow[t]{9}{*}{$36 \mathrm{Kr}$} & $83 m$ & 114 & $M$ & .10134 & -3 & 0 & 0 & 0 & 0 & 0.0096 & 100 & NUS \\
\hline & $85 \mathrm{~m}$ & 4.4 & $\mathrm{H}$ & .43759 & -4 & .5552 & -4 & 0.824 & 81 & $\begin{array}{l}0.15 \\
0.305\end{array}$ & $\begin{array}{l}81 \\
19\end{array}$ & NUS \\
\hline & 85 & 10.6 & $\mathrm{Y}$ & .20721 & -8 & .5552 & -4 & $\begin{array}{l}0.15 \\
0.672\end{array}$ & $\begin{array}{r}1 \\
99\end{array}$ & 0.514 & 1 & NUS \\
\hline & 87 & 78 & $M$ & .14811 & -3 & .328131 & -2 & $\begin{array}{l}1.25 \\
3.3 \\
3.8\end{array}$ & $\begin{array}{l}25 \\
10 \\
65\end{array}$ & $\begin{array}{l}0.403 \\
0.847 \\
2.05 \\
2.57\end{array}$ & $\begin{array}{l}87 \\
13 \\
<3 \\
\overline{2} 2\end{array}$ & NUS \\
\hline & 88 & 2.88 & $\mathrm{H}$ & .66855 & -4 & .869348 & -2 & $\begin{array}{l}0.52 \\
0.9 \\
2.7\end{array}$ & $\begin{array}{l}70 \\
10 \\
20\end{array}$ & $\begin{array}{l}0.028 \\
0.166 \\
0.191 \\
0.36 \\
0.845 \\
1.19 \\
1.55 \\
1.85 \\
2.19 \\
2.40\end{array}$ & $\begin{array}{r}7 \\
7 \\
35 \\
5 \\
23 \\
<4 \\
14 \\
<15 \\
<18 \\
35\end{array}$ & NUS \\
\hline & 89 & 3.16 & $M$ & .36559 & -2 & $\begin{array}{l}.17507 \\
206603\end{array}$ & -1 & $\begin{array}{l}2.0 \\
3.9\end{array}$ & $\begin{array}{l}35 \\
65\end{array}$ & 0 & 0 & NUS \\
\hline & 90 & 33 & $\mathrm{~s}$ & .21005 & -1 & .4418467 & -1 & 3.2 & 100 & 0 & 0 & NUS \\
\hline & 91 & 10 & $s$ & .69315 & -1 & .3846738 & -1 & 1.8 & 100 & 1.8 & 100 & NDS* \\
\hline & 92 & 3 & $S$ & .23105 & -1 & .285014 & -1 & 3.0 & 100 & 3.0 & 100 & NDS* \\
\hline $37 \mathrm{Rb}$ & 87 & $4.3 x$ & ${ }^{0}{ }_{Y}$ & .51080 & -18 & .4282 & -4 & 0.273 & 100 & 0 & 0 & NDS \\
\hline
\end{tabular}




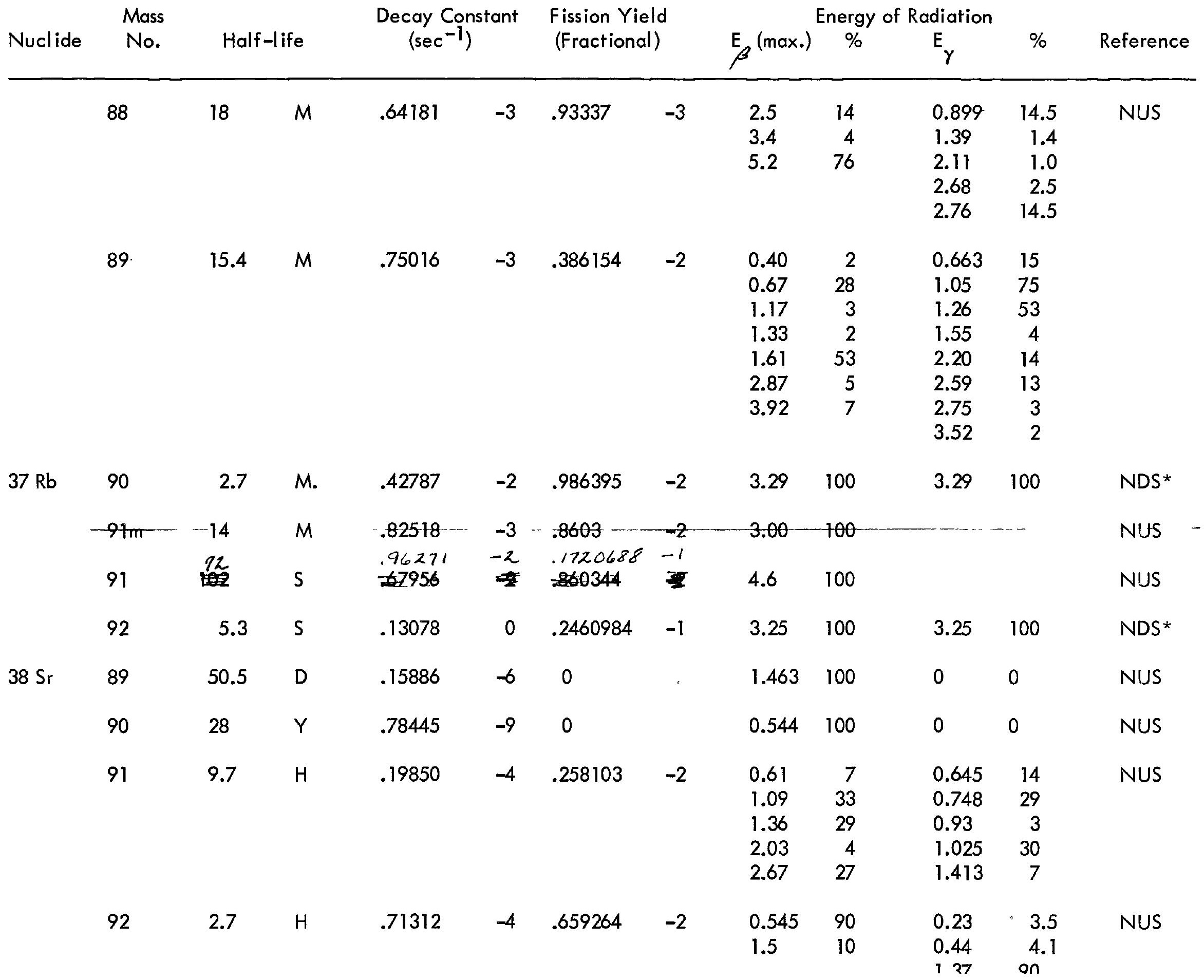




\begin{tabular}{|c|c|c|c|c|c|c|c|c|c|c|c|c|}
\hline \multirow{3}{*}{ Nuclide } & \multirow{3}{*}{$\begin{array}{c}\text { Mass } \\
\text { No. }\end{array}$} & \multirow{2}{*}{\multicolumn{2}{|c|}{ Half-life }} & \multirow{2}{*}{\multicolumn{2}{|c|}{$\begin{array}{l}\text { Decay Constant } \\
\quad\left(\sec ^{-1}\right)\end{array}$}} & \multirow{2}{*}{\multicolumn{2}{|c|}{$\begin{array}{l}\text { Fission Yields } \\
\text { (Fractional) }\end{array}$}} & \multicolumn{4}{|c|}{ Energy of Radiation } & \multirow{3}{*}{$\frac{\text { Reference }}{\text { NDS* }}$} \\
\hline & & & & & & & & $E_{3}(\max )$. & $\%$ & & $\%$ & \\
\hline & & 8.0 & M & .14441 & -2 & .6332533 & -1 & 2.0 & 100 & 2.0 & 100 & \\
\hline & 94 & 1.3 & M & .88865 & -2 & .5940576 & -1 & 1.7 & 100 & 1.7 & 100 & NDS* \\
\hline & 95 & 40 & S & .17329 & -1 & .5294117 & -1 & 2.25 & 100 & 2.25 & 100 & NDS* \\
\hline & 97 & 1.5 & $\mathrm{~s}$ & .46210 & 0 & .613245 & -2 & 3.5 & 100 & 3.5 & 100 & NDS* \\
\hline \multirow[t]{8}{*}{$39 \mathrm{Y}$} & $89 m$ & 16 & $s$ & .43322 & -1 & 0 & & 0 & 0 & 0.91 & 100 & Stehn \\
\hline & 90 & 64.2 & $\mathrm{H}$ & .29991 & -5 & 0 & & 2.27 & 100 & 0 & 0 & NUS \\
\hline & $91 \mathrm{~m}$ & 51 & M & .22652 & -3 & 0 & 0 & 0 & 0 & 0.551 & 100 & NUS \\
\hline & 91 & 58 & D & .13832 & -6 & 0 & & $\begin{array}{l}0.33 \\
1.54\end{array}$ & $100^{0.3}$ & 1.208 & 0.3 & NUS \\
\hline & 92 & 3.5 & $\mathrm{H}$ & .55012 & -4 & .6052 & -4 & $\begin{array}{l}1.26 \\
1.75 \\
3.6\end{array}$ & $\begin{array}{r}9 \\
3 \\
88\end{array}$ & $\begin{array}{l}1.84 \\
2.4\end{array}$ & $\begin{array}{l}3 \\
9\end{array}$ & NUS \\
\hline & 93 & 10.3 & $H$ & .18693 & -4 & .103041 & -2 & $\begin{array}{l}0.45 \\
0.71 \\
1.47 \\
1.95 \\
2.62 \\
2.89\end{array}$ & $\begin{array}{l}0.15 \\
1.8 \\
0.9 \\
3.0 \\
3.9 \\
90\end{array}$ & $\begin{array}{l}0.267 \\
0.49 \\
0.665 \\
0.935 \\
1.15 \\
1.42 \\
1.90 \\
2.41 \\
2.18\end{array}$ & $\begin{array}{l}6.6 \\
0.03 \\
0.7 \\
2.3 \\
0.2 \\
0.7 \\
1.6 \\
0.05 \\
0.3\end{array}$ & NUS \\
\hline & 94 & 20 & $M$ & .57762 & -3 & .445178 & -2 & 5.0 & 40 & $\begin{array}{l}0.56 \\
0.92 \\
1.13\end{array}$ & $\begin{array}{r}6 \\
43 \\
5\end{array}$ & Stehn \\
\hline & 95 & 10 & $M$ & .11552 & -2 & .944378 & -2 & 2.35 & 100 & 2.35 & 100 & NDS* \\
\hline
\end{tabular}




\begin{tabular}{|c|c|c|c|c|c|c|c|c|c|c|c|c|}
\hline \multirow[t]{2}{*}{ Nuclide } & \multirow{2}{*}{$\begin{array}{l}\begin{array}{c}\text { Mass } \\
\text { No. }\end{array} \\
96\end{array}$} & \multicolumn{2}{|c|}{ Half-life } & \multicolumn{2}{|c|}{$\begin{array}{l}\text { Decay Constant } \\
\left(\sec ^{-1}\right)\end{array}$} & \multicolumn{2}{|c|}{$\begin{array}{l}\text { Fission Yield } \\
\text { (Fractional) }\end{array}$} & \multirow[t]{2}{*}{$\mathrm{E}_{\beta}$ (max.) } & \multirow[t]{2}{*}{$\%$} & \multirow[t]{2}{*}{$E_{\gamma}$} & \multirow[t]{2}{*}{$\%$} & \multirow[t]{2}{*}{ Reference } \\
\hline & & 2.3 & $M$ & .50228 & -2 & .602821 & -1 & & & & & \\
\hline $39 Y$ & 97 & 5.0 & $S$ & .13863 & 0 & .2460984 & -1 & 2.75 & 100 & 2.75 & 100 & NDS* \\
\hline \multirow[t]{5}{*}{$40 \mathrm{Zr}$} & 93 & 9.5 & $5^{5} Y$ & .23120 & -13 & 0 & & $\begin{array}{l}0.034 \\
0.063\end{array}$ & $\begin{array}{l}25 \\
75\end{array}$ & 0.029 & 25 & NDS \\
\hline & 95 & 65 & $D$ & .12342 & -6 & .18908 & -3 & $\begin{array}{l}0.360 \\
0.396 \\
0.885 \\
1.13\end{array}$ & $\begin{array}{c}43 \\
55 \\
2 \\
0.4\end{array}$ & $\begin{array}{l}0.235 \\
0.720 \\
0.760\end{array}$ & $\begin{array}{r}2 \\
55 \\
43\end{array}$ & NUS, NDS \\
\hline & 97 & 17.0 & $\mathrm{H}$ & .11326 & -4 & .2410964 & -1 & $\begin{array}{l}0.45 \\
1.91\end{array}$ & $\begin{array}{l}10 \\
90\end{array}$ & $\begin{array}{l}1.02 \\
1.15\end{array}$ & $\begin{array}{l}1 \\
2\end{array}$ & NUS \\
\hline & 98 & 60 & $S$ & .11552 & -1 & .5614245 & -1 & 2.25 & 100 & 2.25 & 100 & NDS* \\
\hline & 99 & $\begin{array}{l}1.6 \\
373\end{array}$ & $S$ & $\begin{array}{r}43323 \\
.005\end{array}$ & $\stackrel{0}{a}$ & .537715 & -1 & 1.8 & 100 & 1.8 & 100 & NDS* \\
\hline \multirow[t]{6}{*}{$41 \mathrm{Nb}$} & $93 m$ & 3.7 & $Y$ & .59364 & -8 & 0 & & 0 & 0 & 0.029 & 100 & NDS \\
\hline & $95 m$ & 90 & $H$ & .21394 & -5 & 0 & & 0 & 0 & 0.235 & 100 & NUS \\
\hline & 95 & 35 & $D$ & .22922 & -6 & 0 & & $\begin{array}{l}0.160 \\
0.93\end{array}$ & $\begin{array}{r}99 \\
1\end{array}$ & 0.762 & 99 & NUS \\
\hline & $97 m$ & 60 & $S$ & .11552 & -1 & 297619 & -2 & 0 & 0 & 0.75 & 100 & NUS \\
\hline & 97 & 73 & $M$ & .15825 & -3 & .297619 & -2 & $\begin{array}{l}0.93 \\
1.267\end{array}$ & $\begin{array}{r}1 \\
99\end{array}$ & $\begin{array}{l}0.665 \\
1.02\end{array}$ & $\begin{array}{r}99 \\
1\end{array}$ & NUS \\
\hline & $98 m$ & 51 & $M$ & .22652 & -3 & .860345 & -3 & 3.1 & 41 & $\begin{array}{l}0.72 \\
0.78 \\
1.16 \\
1.68 \\
1.88\end{array}$ & $\begin{array}{r}75 \\
100 \\
30 \\
10 \\
4\end{array}$ & NUS \\
\hline
\end{tabular}




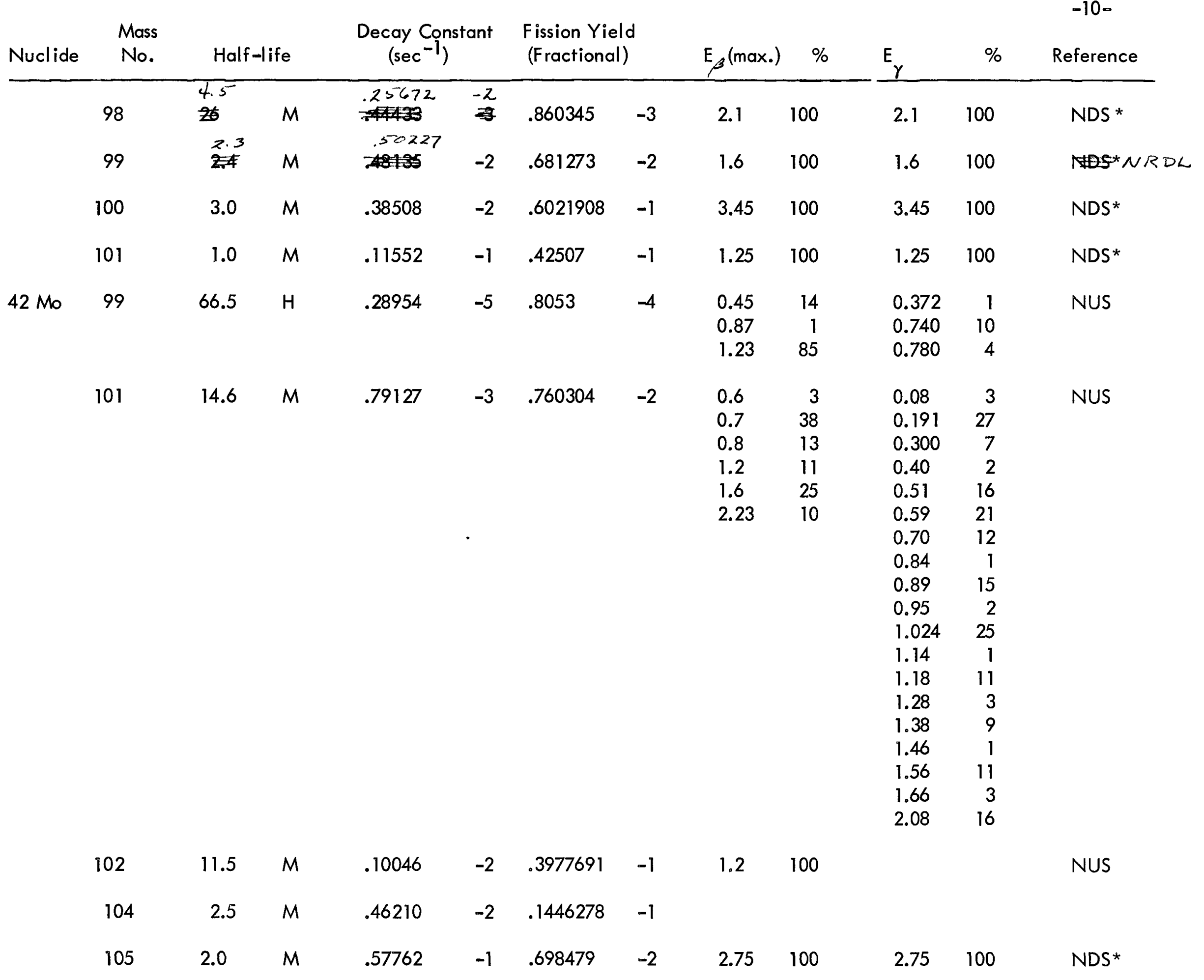




\begin{tabular}{|c|c|c|c|c|c|c|c|c|c|c|c|c|}
\hline \multirow{2}{*}{$\frac{\text { Nuclide }}{43 \mathrm{Tc}}$} & \multirow{2}{*}{$\begin{array}{l}\text { Mass } \\
\text { No. }\end{array}$} & \multicolumn{2}{|c|}{ Half-life } & \multicolumn{2}{|c|}{$\begin{array}{l}\text { Decay Constant } \\
\quad\left(\sec ^{-1}\right)\end{array}$} & \multicolumn{2}{|c|}{$\begin{array}{l}\text { Fission Yield } \\
\text { (Fractional) }\end{array}$} & \multirow{2}{*}{$\frac{\mathrm{E}_{\beta}(\max .)}{0}$} & \multirow{2}{*}{$\frac{\%}{0}$} & \multirow{2}{*}{\multicolumn{2}{|c|}{$\begin{array}{c}-11- \\
\% \\
98.6 \\
98.6 \\
1.4\end{array}$}} & \multirow{2}{*}{$\begin{array}{c}\text { Reference } \\
\text { NUS }\end{array}$} \\
\hline & & 6.0 & $\mathrm{H}$ & .32090 & -4 & 0 & & & & & & \\
\hline & 99 & \multicolumn{2}{|c|}{$2.1 \times 10^{5} Y$} & .10459 & -12 & 0 & & 0.292 & 100 & 0 & 0 & \\
\hline & 101 & 14.0 & $M$ & .82518 & -3 & .15706 & -3 & $\begin{array}{l}1.07 \\
1.32\end{array}$ & $\begin{array}{r}8 \\
90\end{array}$ & $\begin{array}{l}0.307 \\
0.543\end{array}$ & $\begin{array}{r}90 \\
8\end{array}$ & \\
\hline & $102 \mathrm{~m}$ & 4.5 & $M$ & .25672 & -2 & .70528 & -3 & 2 & 100 & 2.1 & 100 & NDS* \\
\hline & 102 & 5 & $S$ & .13863 & 0 & .70528 & -3 & 2.05 & 100 & 2.05 & 100 & NDS* \\
\hline & 103 & 72 & $S$ & .96271 & -2 & .297891 & -1 & 1.25 & 100 & 1.25 & 100 & NDS* \\
\hline & 104 & 18 & $M$ & .64181 & -3 & .340136 & -2 & 2.4 & 100 & 2.6 & 100 & NDS* \\
\hline & 105 & 9.0 & $M$ & .12836 & -2 & .198079 & -2 & 1.7 & 100 & 1.7 & 100 & NDS* \\
\hline & 107 & 60 & $S$ & .11552 & -1 & .182703 & -2 & 3 & 100 & 3 & 100 & NDS* \\
\hline \multirow[t]{3}{*}{$44 R u$} & 103 & 39.7 & $D$ & .20208 & -6 & 0 & & $\begin{array}{l}0.10 \\
0.212 \\
0.39 \\
0.71\end{array}$ & $\begin{array}{r}7 \\
89 \\
1 \\
3\end{array}$ & $\begin{array}{l}0.323 \\
0.498 \\
0.610\end{array}$ & $\begin{array}{r}3 \\
89 \\
7\end{array}$ & NIJS \\
\hline & 105 & 4.45 & $H$ & .43268 & -4 & .7763 & -4 & $\begin{array}{l}0.526 \\
0.915 \\
1.080 \\
1.145 \\
1.87\end{array}$ & $\begin{array}{r}4 \\
8 \\
30 \\
48 \\
10\end{array}$ & $\begin{array}{l}0.263 \\
0.318 \\
0.400 \\
0.475 \\
0.485 \\
0.670 \\
0.726 \\
0.874 \\
0.966\end{array}$ & $\begin{array}{c}5 \\
10.6 \\
5.6 \\
16 \\
5 \\
15 \\
48 \\
4.3 \\
3\end{array}$ & N'JS \\
\hline & 106 & 1.00 & $Y$ & .21965 & -7 & .392246 & -2 & 0.039 & 100 & 0 & 0 & NUS \\
\hline
\end{tabular}




\begin{tabular}{|c|c|c|c|c|c|c|c|c|c|c|c|c|}
\hline \multirow[t]{2}{*}{ Nuclide } & \multirow{2}{*}{$\begin{array}{l}\text { Mass } \\
\text { No. } \\
107\end{array}$} & \multicolumn{2}{|c|}{ Half-life } & \multicolumn{2}{|c|}{$\begin{array}{l}\text { Decay Constant } \\
\left(\sec ^{-1}\right)\end{array}$} & \multicolumn{2}{|c|}{$\begin{array}{l}\text { Fission Yield } \\
\text { (Fractional) }\end{array}$} & $\frac{E_{\beta}(\max .)}{3.0}$ & $\frac{\%}{12}$ & \multirow{2}{*}{$\begin{array}{l}E_{\gamma} \\
0.195 \\
0.86 \\
1.03\end{array}$} & \multirow{2}{*}{$\begin{array}{r}\% \\
17 \\
8 \\
4\end{array}$} & \multirow{2}{*}{$\frac{\begin{array}{c}-12- \\
\text { Reference }\end{array}}{\text { NUS }}$} \\
\hline & & 4.6 & $M$ & .25114 & -2 & .8063 & -4 & $\begin{array}{r}3.0 \\
3.8 \\
4\end{array}$ & $\begin{array}{r}12 \\
9 \\
79\end{array}$ & & & \\
\hline & 108 & 4.3 & $M$ & .26866 & -2 & .70038 & -3 & $\begin{array}{l}1.15 \\
1.32\end{array}$ & $\begin{array}{l}28 \\
72\end{array}$ & 0.165 & 28 & NDS \\
\hline \multirow[t]{9}{*}{$45 \mathrm{Rh}$} & $103 \mathrm{~m}$ & 57 & $M$ & .20268 & -3 & 0 . & & 0 & 0 & 0.040 & 100 & NUS \\
\hline & $105 m$ & 38 & $S$ & .18241 & -1 & 0 & & 0 & 0 & 0.129 & 100 & N'JS \\
\hline & 105 & 36 & $\mathrm{H}$ & .53484 & -5 & 0 & & $\begin{array}{r}0.25 \\
0.565\end{array}$ & $\begin{array}{l}10 \\
90\end{array}$ & 0.319 & 10 & N'JS \\
\hline & 106 & 30 & $S$ & .23105 & -1 & 0 & & $\begin{array}{l}2.0 \\
2.4 \\
3.0 \\
3.54\end{array}$ & $\begin{array}{r}2 \\
11 \\
8 \\
78\end{array}$ & $\begin{array}{l}0.513 \\
0.612 \\
0.624 \\
1.045\end{array}$ & $\begin{array}{c}20.5 \\
1 \\
9.9 \\
1.5\end{array}$ & N'JS \\
\hline & 107 & 22 & $M$ & .52511 & -3 & 0 & & $\begin{array}{l}0.9 \\
1.1 \\
1.2\end{array}$ & $\begin{array}{r}3 \\
13 \\
84\end{array}$ & $\begin{array}{l}0.305 \\
0.39 \\
0.68\end{array}$ & $\begin{array}{r}84 \\
13 \\
3\end{array}$ & NUS \\
\hline & 108 & 18 & $\mathrm{~S}$ & .38508 & -1 & 0 & & $\begin{array}{l}3.5 \\
4.1 \\
4.5\end{array}$ & $\begin{array}{l}22 \\
17 \\
51\end{array}$ & $\begin{array}{l}0.433 \\
0.617\end{array}$ & $\begin{array}{l}43 \\
22\end{array}$ & NDS \\
\hline & $109 \mathrm{~m}$ & 50 & $S$ & .13863 & -1 & .150385 & -3 & 0 & 0 & 0.11 & 100 & NDS \\
\hline & 109 & 30 & $S$ & .23105 & -1 & .150385 & -3 & 1.4 & 100 & 1.4 & 100 & NDS* \\
\hline & 110 & 3.6 & $S$ & .19254 & 0 & .17776 & -3 & & & & & \\
\hline \multirow[t]{2}{*}{$46 \mathrm{Pd}$} & 107 & $7 \times 10^{6}$ & $Y$ & .31378 & -14 & 0 & & 0.035 & 100 & 0 & 0 & NDS \\
\hline & $109 \mathrm{~m}$ & 4.7 & $M$ & .24580 & -2 & 0 & & 0 & 0 & 0.18 & 100 & NDS \\
\hline
\end{tabular}




\begin{tabular}{|c|c|c|c|c|c|c|c|c|c|c|c|c|}
\hline \multirow{2}{*}{$\frac{\text { Nuclide }}{10}$} & \multirow{2}{*}{$\begin{array}{r}\begin{array}{r}\text { Mass } \\
\text { No. }\end{array} \\
109\end{array}$} & \multicolumn{2}{|c|}{ Half-life } & \multicolumn{2}{|c|}{$\begin{array}{l}\text { Decay Constant } \\
\quad\left(\sec ^{-1}\right)\end{array}$} & \multicolumn{2}{|c|}{$\begin{array}{l}\text { Fission Yield } \\
\text { (Fractional) }\end{array}$} & \multirow{2}{*}{$\frac{\mathrm{E}_{\beta}(\max .)}{1.025}$} & \multirow{2}{*}{$\frac{\%}{100}$} & \multirow{2}{*}{$\frac{E_{\gamma}}{0}$} & \multirow{2}{*}{$\frac{\begin{array}{c}-13- \\
\%\end{array}}{0}$} & \multirow{2}{*}{$\begin{array}{c}\text { Reference } \\
\text { NUS }\end{array}$} \\
\hline & & 14 & $\mathrm{H}$ & .13753 & -4 & 0 & & & & & & \\
\hline & $111 \mathrm{~m}$ & 5.5 & $\mathrm{H}$ & .35008 & -4 & .2080 & -5 & 0.61 & 32 & 0.17 & 68 & NDS \\
\hline & 111 & 23 & $M$ & .50228 & -3 & .17884 & -3 & 2.13 & 100 & 0 & 0 & NUS \\
\hline & 112 & 21 & $\mathrm{H}$ & .91687 & -5 & .10008 & -3 & 0.28 & 100 & 0.018 & 100 & NUS \\
\hline & 113 & 84 & $S$ & .82518 & -2 & .16048 & -3 & 1.65 & 100 & 1.65 & 100 & NDS* \\
\hline & 114 & 2.4 & $M$ & .48135 & -2 & .1355 & -3 & 1.4 & 100 & 0 & 0 & NDS \\
\hline & 115 & 45 & $S$ & .15403 & -1 & .10285 & -3 & 2.25 & 100 & 2.25 & 100 & NDS* \\
\hline \multirow[t]{6}{*}{$47 \mathrm{Ag}$} & $109 \mathrm{~m}$ & 39 & $S$ & .17773 & -1 & 0 & 0 & 0 & 0 & 0.088 & 100 & NUS \\
\hline & $111 \mathrm{~m}$ & 74 & $S$ & .93669 & -2 & 0 & 0 & 0 & 0 & 0.065 & 100 & NDS \\
\hline & 111 & 7.6 & D & .10556 & -5 & 0 & & $\begin{array}{l}0.69 \\
0.79 \\
1.05\end{array}$ & $\begin{array}{r}6.2 \\
1.1 \\
92.7\end{array}$ & $\begin{array}{l}0.095 \\
0.247 \\
0.342\end{array}$ & $\begin{array}{l}0.1 \\
1.2 \\
6.1\end{array}$ & NUS \\
\hline & 112 & 3.12 & $H$ & .61712 & -4 & 0 & & $\begin{array}{l}1.22 \\
1.34 \\
1.50 \\
1.61 \\
1.78 \\
2.01 \\
2.20 \\
2.57 \\
2.63 \\
2.73 \\
3.42 \\
4.04\end{array}$ & $\begin{array}{c}2.6 \\
3.6 \\
1.8 \\
2.0 \\
4.4 \\
5.3 \\
0.6 \\
1.6 \\
0.9 \\
2.8 \\
18 \\
56\end{array}$ & $\begin{array}{l}0.615 \\
0.69 \\
0.855 \\
0.98 \\
1.09 \\
1.31 \\
1.40 \\
1.49 \\
1.62 \\
2.11 \\
2.54\end{array}$ & $\begin{array}{l}6.5 \\
4.5 \\
1.6 \\
1.2 \\
1.2 \\
1.4 \\
5.3 \\
1.2 \\
3.2 \\
3.2 \\
1.0\end{array}$ & NUS \\
\hline & $113 \mathrm{~m}$ & 72 & $S$ & .96271 & -2 & 0 & & $<2$ & 10 & 0.3 & 90 & NDS \\
\hline & 113 & 5.3 & $H$ & .36329 & -4 & 0 & & 1.7 & $\mathrm{Ims}$ & 2 & 7 & NOS \\
\hline
\end{tabular}




\begin{tabular}{|c|c|c|c|c|c|c|c|c|c|c|c|c|}
\hline \multirow[t]{2}{*}{ Nuclide } & \multirow{2}{*}{$\begin{array}{c}\begin{array}{c}\text { Mass } \\
\text { No. }\end{array} \\
114\end{array}$} & \multicolumn{2}{|c|}{ Half-life } & \multicolumn{2}{|c|}{$\begin{array}{l}\text { Decay Constant } \\
\qquad\left(\sec ^{-1}\right)\end{array}$} & \multicolumn{2}{|c|}{$\begin{array}{l}\text { Fission Yield } \\
\text { (Fractional) }\end{array}$} & \multirow{2}{*}{$\frac{\mathrm{E}_{\beta}(\max )}{2.3}$} & \multirow{2}{*}{$\frac{\%}{100}$} & \multirow{2}{*}{$\frac{\mathrm{E}_{\gamma}}{2.3}$} & \multirow{2}{*}{$\frac{\%}{100}$} & \multirow{2}{*}{$\frac{\text { Reference }}{\text { NDS* }}$} \\
\hline & & 5.0 & $S$ & .13863 & 0 & .1860 & -5 & & & & & \\
\hline & $115 \mathrm{~m}$ & 20 & $\mathrm{~S}$ & .34657 & -1 & .3530 & -5 & 2.9 & 28 & 1.2 & 72 & NDS \\
\hline & 115 & 21 & $M$ & .55012 & -3 & .3530 & -5 & $\begin{array}{l}2.7 \\
2.9\end{array}$ & $\begin{array}{r}9 \\
91\end{array}$ & $\begin{array}{l}0.138 \\
0.227\end{array}$ & $\begin{array}{l}\text { Weak } \\
\text { Weak }\end{array}$ & NDS \\
\hline & 116 & 2.5 & $M$ & .46210 & -2 & $\begin{array}{l}.18525 \\
.026\end{array}$ & -3 & 5.0 & 100 & $\begin{array}{l}0.52 \\
0.70\end{array}$ & $\begin{array}{l}100 \\
100\end{array}$ & NDS \\
\hline & 117 & 66 & $S$ & .10502 & -1 & .10614 & -3 & 1.9 & 100 & 1.9 & 100 & NDS* \\
\hline \multirow[t]{8}{*}{$48 \mathrm{Cd}$} & $115 \mathrm{~m}$ & 43 & $D$ & .18567 & -6 & 0 & & $\begin{array}{l}0.335 \\
0.687 \\
1.63\end{array}$ & $\begin{array}{r}1 \\
2 \\
97\end{array}$ & $\begin{array}{l}0.48 \\
0.935 \\
1.3\end{array}$ & $\begin{array}{l}0.3 \\
2.3 \\
1\end{array}$ & NDS \\
\hline & 115 & 53 & $\mathrm{H}$ & .36329 & -5 & 0 & & $\begin{array}{l}0.59 \\
0.63 \\
0.85 \\
1.11\end{array}$ & $\begin{array}{r}24.4 \\
12.6 \\
0.9 \\
62.1\end{array}$ & $\begin{array}{l}0.26 \\
0.49 \\
0.523\end{array}$ & $\begin{array}{l}1.7 \\
12.2 \\
24\end{array}$ & NUS \\
\hline & $117 m$ & 3.0 & $\mathrm{H}$ & .64181 & -4 & .1940 & -5 & 1.0 & $<100$ & $\begin{array}{l}0.28 \\
1.27\end{array}$ & $\begin{array}{l}100 \\
100\end{array}$ & NDS \\
\hline & 117 & 50 & $M$ & .23105 & -3 & .1940 & -5 & 2.3 & $<100$ & 0.425 & 100 & NUS \\
\hline & 118 & 50 & $M$ & .23105 & -3 & .14236 & -3 & 0.8 & 100 & 0 & 0 & NUS \\
\hline & 119 & 9.5 & $M$ & $\begin{array}{l}12160 \\
\quad 502\end{array}$ & -2 & .7013 & -4 & $\begin{array}{l}1.8 \\
3.5\end{array}$ & $\begin{array}{l}20 \\
80\end{array}$ & 1.8 & 20 & NDS \\
\hline & $119 \mathrm{~m}$ & $\begin{array}{l}2.7 \\
\vec{z}\end{array}$ & $M$ & $\begin{array}{r}42787 \\
-508\end{array}$ & -2 & .7013 & -4 & 1.85 & 100 & 1.85 & 100 & NDS* \\
\hline & 121 & 25 & $S$ & .27726 & -1 & .12005 & -3 & 2.4 & 100 & 2.4 & 100 & NDS* \\
\hline $49 \ln$ & $115 \mathrm{~m}$ & 4.51 & $H$ & .42692 & -4 & 0 & & 0.84 & 5.5 & 0.335 & 94.5 & NUS \\
\hline
\end{tabular}




\begin{tabular}{|c|c|c|c|c|c|c|c|c|c|c|c|c|}
\hline \multirow[t]{2}{*}{ Nuclide } & \multirow{2}{*}{$\begin{array}{c}\begin{array}{c}\text { Mass } \\
\text { No. }\end{array} \\
115\end{array}$} & \multicolumn{2}{|c|}{ Halfolife } & \multicolumn{2}{|c|}{$\begin{array}{l}\text { Decay Constant } \\
\left(\sec ^{-1}\right)\end{array}$} & \multicolumn{2}{|c|}{$\begin{array}{l}\text { Fission Yield } \\
\text { (Fractional) }\end{array}$} & \multirow{2}{*}{$\frac{E_{\beta} \text { (max.) }}{0.50}$} & \multirow{2}{*}{$\frac{\%}{100}$} & \multicolumn{2}{|c|}{$\begin{array}{c}-15- \\
\%\end{array}$} & \multirow{2}{*}{$\frac{\text { Release }}{\text { NDS }}$} \\
\hline & & $6 \times 10^{4}$ & $Y$ & .36608 & -22 & 0 & & & & 0 & 0 & \\
\hline & $117 \mathrm{~m}$ & 2.0 & $\mathrm{H}$ & .96271 & -4 & 0 & & $\begin{array}{l}0.95 \\
1.62 \\
1.77\end{array}$ & $\begin{array}{r}4 \\
23 \\
53\end{array}$ & $\begin{array}{l}0.161 \\
0.31 \\
0.822\end{array}$ & $\begin{array}{r}23 \\
20 \\
4\end{array}$ & NDS \\
\hline & 117 & 45 & $M$ & .25672 & -3 & 0 & & 0.740 & 100 & $\begin{array}{l}0.161 \\
0.565\end{array}$ & $\begin{array}{l}100 \\
100\end{array}$ & NDS \\
\hline & 118 & 5.0 & $S$ & .13863 & 0 & 0 & & $\begin{array}{l}3.3 \\
4.5\end{array}$ & $\begin{array}{l}20 \\
80\end{array}$ & 1.22 & 20 & NDS \\
\hline & $119 m$ & 18 & $M$ & .64181 & -3 & .7500 & -6 & $\begin{array}{l}1.8 \\
2.7\end{array}$ & $\begin{array}{r}6 \\
90\end{array}$ & $\begin{array}{l}0.30 \\
0.907\end{array}$ & $\begin{array}{l}4 \\
6\end{array}$ & NDS \\
\hline & 119 & 2.3 & $M$ & .50228 & -2 & .7500 & -6 & 1.6 & 100 & $\begin{array}{l}0.024 \\
0.82\end{array}$ & $\begin{array}{l}<90 \\
<90\end{array}$ & NDS \\
\hline & 120 & 50 & $S$ & .13863 & -1 & .14091 & -3 & 2.2 & 100 & $\begin{array}{l}0.089 \\
0.199 \\
1.04 \\
1.18\end{array}$ & $\begin{array}{l}100 \\
100 \\
100 \\
100\end{array}$ & NDS \\
\hline & $121 \mathrm{~m}$ & 3.1 & $M$ & .37266 & -2 & .10105 & -4 & 1.85 & 100 & 1.85 & 100 & NDS* \\
\hline & 121 & 30 & $s$ & .23105 & -1 & .10105 & -4 & 2.9 & 100 & 0.94 & 100 & NDS \\
\hline & 122 & 45 & $S$ & .15403 & -1 & .14366 & -3 & 3 & 100 & 3 & 100 & $\underset{0}{\mathrm{NDS}}$ * \\
\hline & $123 m$ & 36 . & $S$ & .19254 & -1 & .73135 & -4 & 2.3 & 100 & 2.3 & 100 & NDS* \\
\hline & 123 & 10 & $S$ & .69315 & -1 & .73135 & -4 & 3.3 & 100 & 1.10 & 100 & NDS \\
\hline & 124 & 20 & $S$ & .34657 & -1 & .14175 & -3 & & & & & \\
\hline
\end{tabular}




\begin{tabular}{|c|c|c|c|c|c|c|c|c|c|c|c|c|}
\hline \multirow{2}{*}{$\begin{array}{l}\text { Nuclide } \\
50 \mathrm{Sn}\end{array}$} & \multirow{2}{*}{$\begin{array}{c}\begin{array}{c}\text { Mass } \\
\text { No. }\end{array} \\
117 \mathrm{~m}\end{array}$} & \multicolumn{2}{|c|}{ Half-life } & \multicolumn{2}{|c|}{$\begin{array}{l}\text { Decay Constant } \\
\qquad\left(\sec ^{-1}\right)\end{array}$} & \multicolumn{2}{|c|}{$\begin{array}{l}\text { Fission Yield } \\
\text { (Fractional) }\end{array}$} & $E_{\beta}(\max )$. & $\%$ & $E_{\gamma}$ & $\begin{array}{c}-16= \\
\%\end{array}$ & Reference \\
\hline & & 14 & $D$ & .57304 & -6 & 0 & & 0 & 0 & 0.159 & 100 & NDS \\
\hline & $119 \mathrm{~m}$ & 245 & $D$ & .32738 & -7 & 0 & & 0 & 0 & $\begin{array}{l}0.161 \\
0.0242 \\
0.0653\end{array}$ & $\begin{array}{l}100 \\
100 \\
100\end{array}$ & Nos \\
\hline & $121 \mathrm{~m}$ & 5.0 & $Y$ & .43929 & -8 & .1700 & -6 & 0.42 & 100 & 0.01 & 100 & NDS \\
\hline & 121 & 27.0 & $H$ & .71312 & -5 & .1700 & -6 & 0.383 & 100 & 0 & 0 & NUS \\
\hline & $123 \mathrm{~m}$ & 125 & D & .64181 & -7 & .7605 & -5 & $\begin{array}{l}0.34 \\
1.42\end{array}$ & $\begin{array}{r}2 \\
98\end{array}$ & 1.08 & 2 & N'JS \\
\hline & 123 & 41 & M. & .28177 & -3 & .7605 & -5 & 1.26 & 100 & 0.16 & 100 & NISS \\
\hline & $125 m$ & 9.7 & $M$ & .11910 & -2 & .10169 & -3 & $\begin{array}{l}0.65 \\
2.04\end{array}$ & $\begin{array}{r}2.2 \\
97.8\end{array}$ & $\begin{array}{l}0.326 \\
0.64 \\
1.068 \\
1.39\end{array}$ & $\begin{array}{r}99.7 \\
0.3 \\
0.3 \\
1.9\end{array}$ & NUS \\
\hline & 125 & 9.4 & $D$ & .85346 & -6 & .10169 & -3 & $\begin{array}{l}0.37 \\
0.47 \\
1.3 \\
2.33\end{array}$ & $\begin{array}{l}2.1 \\
1.5 \\
1.3 \\
95\end{array}$ & $\begin{array}{l}8.468 \\
\$ .811 \\
0.904 \\
1.068 \\
1.97\end{array}$ & $\begin{array}{l}0.5 \\
1.0 \\
0.9 \\
3.9 \\
1.2\end{array}$ & NIJS \\
\hline & 126 & $2 \times 10^{5}$ & $Y$ & .10982 & -12 & .27472 & -3 & 0.4 & 100 & & & NDS \\
\hline & 127 & $\frac{2.6}{20}$ & $H$ & $\begin{array}{l}.644231 \\
.96273\end{array}$ & -4 & .106743 & -2 & 1.6 & 100 & 1.6 & 100 & NDS* \\
\hline & 128 & 57 & $M$ & .20268 & -3 & .238096 & -2 & 1.3 & 100 & 0 & 0 & NUS \\
\hline & 129 & 1.8 & $\mathrm{H}$ & .10697 & -3 & .449309 & -2 & 2.0 & 100 & 2.0 & 100 & NDS* \\
\hline & 130 & 2.6 & $M$ & .44433 & -2 & .953381 & -2 & 1.0 & 100 & 1.0 & 100 & NDS* \\
\hline & 131 & 3.4 & $M$ & .33978 & -2 & .1151461 & -1 & 2.5 & 100 & 2.5 & 100 & NDS* \\
\hline & 132 & 2.2 & $M$ & .52511 & -2 & .931972 & -2 & 1.5 & 100 & 1.5 & 100 & NDS* \\
\hline
\end{tabular}




\begin{tabular}{|c|c|c|c|c|c|c|c|c|c|c|c|c|}
\hline \multirow{2}{*}{$\begin{array}{l}\text { Nuclide } \\
51 \mathrm{Sb}\end{array}$} & \multirow{2}{*}{$\begin{array}{l}\begin{array}{l}\text { Mass } \\
\text { No. }\end{array} \\
124 \mathrm{~mm}\end{array}$} & \multicolumn{2}{|c|}{ Half-life } & \multicolumn{2}{|c|}{$\begin{array}{l}\text { Decay Constant } \\
\qquad\left(\sec ^{-1}\right)\end{array}$} & \multicolumn{2}{|c|}{$\begin{array}{l}\text { Fission Yield } \\
\text { (Fractional) }\end{array}$} & \multirow{2}{*}{$\frac{E_{\beta}(\max .)}{2.5}$} & $\%$ & \multirow[t]{2}{*}{$\mathrm{E}_{\gamma}$} & \multirow[t]{2}{*}{$\begin{array}{c}-17= \\
\%\end{array}$} & \multirow{2}{*}{$\frac{\text { Reference }}{\text { VDS }}$} \\
\hline & & 21 & $M$ & .55012 & -3 & .566667 & -6 & & $<100$ & & & \\
\hline & $124 m$ & 1.3 & $M$ & .88865 & -2 & .566667 & -6 & 3.2 & 100 & 0.012 & Small & NDS, Stehn \\
\hline & 124 & 60 & $D$ & . 13371 & -6 & .566667 & -6 & $\begin{array}{l}0.051 \\
0.225 \\
0.621 \\
0.954 \\
1.016 \\
1.59 \\
2.313\end{array}$ & $\begin{array}{c}2 \\
11 \\
51 \\
5 \\
1.5 \\
5 \\
23\end{array}$ & $\begin{array}{l}0.603 \\
0.645 \\
0.722 \\
0.970 \\
1.05 \\
1.298 \\
1.32 \\
1.361 \\
1.37 \\
1.45 \\
1.50 \\
1.54 \\
1.69 \\
1.900 \\
2.09 \\
2.26\end{array}$ & $\begin{array}{c}98 \\
7.2 \\
10.5 \\
2 \\
2 \\
1.2 \\
2 \\
1 \\
2.5 \\
2.0 \\
7 \\
48 \\
48 \\
0.4 \\
6.3 \\
1.5\end{array}$ & $\begin{array}{l}\text { NDS } \\
\\
\\
.85 \\
.85-\end{array}$ \\
\hline & 125 & 2.0 & $\mathrm{Y}$ & . 10982 & -7 & .1281 & -4 & $\begin{array}{l}0.09 \\
0.105 \\
0.118 \\
0.124 \\
0.233 \\
0.295 \\
0.437 \\
0.612\end{array}$ & $\begin{array}{c}1.9 \\
<0.7 \\
6.0 \\
30.7 \\
1 \\
40.4 \\
5.8 \\
13.4\end{array}$ & $\begin{array}{l}0.109 \\
0.171 \\
0.176 \\
0.203 \\
0.206 \\
0.319 \\
0.379 \\
0.427 \\
0.462 \\
0.598 \\
0.604 \\
0.633 \\
0.652 \\
0.668\end{array}$ & $\begin{array}{c}0.4 \\
0.3 \\
6.8 \\
0.2 \\
0.3 \\
0.8 \\
1.2 \\
31 \\
10 \\
19 \\
5.2 \\
11 \\
<0.7 \\
1.6\end{array}$ & NIJS \\
\hline
\end{tabular}

$\begin{array}{lllllllllll}126 \mathrm{~m} & 19 & M & .60803 & -3 & .2196 & -4 & 3.7 & 1 & 0.1 & 99\end{array}$




\begin{tabular}{|c|c|c|c|c|c|c|c|c|c|c|c|c|}
\hline \multirow[t]{2}{*}{ Nuclide } & \multirow{2}{*}{$\begin{array}{l}\text { Mass } \\
\text { No. }\end{array}$} & \multicolumn{2}{|c|}{ Half-life } & \multicolumn{2}{|c|}{$\begin{array}{l}\text { Decay Constant } \\
\qquad\left(\sec ^{-1}\right)\end{array}$} & \multicolumn{2}{|c|}{$\begin{array}{l}\text { Fission Yield } \\
\text { (Fractional) }\end{array}$} & \multirow{2}{*}{$\frac{E_{\beta} \text { (max.) }}{1.9}$} & \multirow{2}{*}{$\frac{\%}{100}$} & \multirow{2}{*}{ 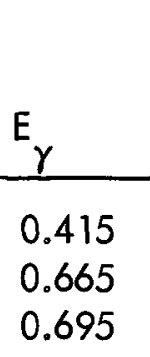 } & \multirow{2}{*}{$\begin{array}{c}-18- \\
\% \\
100 \\
100 \\
100\end{array}$} & \multirow{2}{*}{$\frac{\text { Reference }}{\text { NDS }}$} \\
\hline & & 12.5 & D & .64181 & -6 & .2196 & -4 & & & & & \\
\hline & 127 & 91 & $H$ & .21158 & -5 & .20708 & -3 & $\begin{array}{l}0.8 \\
0.86 \\
1.11 \\
1.50\end{array}$ & $\begin{array}{l}35 \\
10 \\
35 \\
20\end{array}$ & $\begin{array}{l}0.06 \\
0.25 \\
0.31 \\
0.46 \\
0.77\end{array}$ & $\begin{array}{r}4 \\
14 \\
6 \\
54 \\
24\end{array}$ & NUS \\
\hline & $128 \mathrm{~m}$ & $\begin{array}{l}11.0 \\
1.3\end{array}$ & M & $\begin{array}{l}10500 \\
1216\end{array}$ & -2 & .585235 & -3 & 1.0 & 100 & 0 & 0 & NUS \\
\hline & 128 & $\begin{array}{l}8.6 \\
8.8\end{array}$ & $\mathrm{H}$ & $\begin{array}{r}22384 \\
747\end{array}$ & -4 & .585235 & -3 & 1.45 & 100 & 1.45 & 100 & NDS* \\
\hline & 129 & 4.6 & $\mathrm{H}$ & .41857 & -4 & .291116 & -2 & $\begin{array}{l}1.0 \\
1.87\end{array}$ & $\begin{array}{l}80 \\
20\end{array}$ & $\begin{array}{l}0.534 \\
0.165 \\
0.308 \\
0.788\end{array}$ & $\begin{array}{l}20 \\
26.7 \\
26.7 \\
26.7\end{array}$ & $\begin{array}{l}\text { NUS } \\
7 \\
7\end{array}$ \\
\hline & 130 & 7.1 & M & .16271 & -2 & .812325 & -2 & 2.5 & 100 & 2.5 & 100 & NDS* \\
\hline & 131 & 23 & M & .50228 & -3 & .1310524 & -1 & 1.6 & 100 & 1.6 & 100 & NDS* \\
\hline & 132 & 2.1 & M & .55012 & -2 & .2060824 & -1 & 3 & 100 & 3 & 100 & NDS* \\
\hline & 133 & 4.1 & M & .28177 & -2 & .332533 & -1 & 2 & 100 & 2 & 100 & NDS* \\
\hline & 134 & 48 & $s$ & .14441 & -1 & .1805722 & -1 & 4.3 & 100 & 4.3 & 100 & NUS* \\
\hline & 135 & 6.0 & $s$ & .11552 & 0 & .437175 & -2 & 3.5 & 100 & 3.5 & 100 & NDS* \\
\hline \multirow[t]{2}{*}{$52 \mathrm{Te}$} & $125 \mathrm{~m}$ & 58 & D & .13832 & -6 & 0 & & 0 & 0 & $\begin{array}{l}0.0353 \\
0.110\end{array}$ & $\begin{array}{l}100 \\
100\end{array}$ & NDS \\
\hline & $127 \mathrm{~m}$ & 105 & D & .76405 & -7 & .13005 & -4 & $\begin{array}{l}0.054 \\
0.73\end{array}$ & $\begin{array}{l}0.013 \\
1.5\end{array}$ & $\begin{array}{l}0.059 \\
0.0887 \\
0.665\end{array}$ & $\begin{array}{c}1.5 \\
98.5 \\
0.013\end{array}$ & NDS \\
\hline
\end{tabular}




\begin{tabular}{|c|c|c|c|c|c|c|c|c|c|c|c|c|}
\hline \multirow[t]{2}{*}{ Nuclide } & \multirow{2}{*}{$\begin{array}{c}\begin{array}{c}\text { Mass } \\
\text { No. }\end{array} \\
127\end{array}$} & \multicolumn{2}{|c|}{ Half - life } & \multicolumn{2}{|c|}{$\begin{array}{c}\text { Decay Constant } \\
\left(\sec ^{-1}\right)\end{array}$} & \multicolumn{2}{|c|}{$\begin{array}{l}\text { Fission Yield } \\
\text { (Fractional) }\end{array}$} & \multirow{2}{*}{$\frac{E_{\beta}(\max )}{\begin{array}{l}0.27 \\
0.695\end{array}}$} & \multirow{2}{*}{ 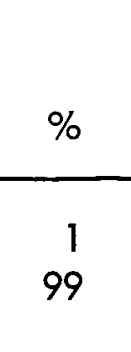 } & \multicolumn{2}{|r|}{$\begin{array}{c}-19- \\
\%\end{array}$} & \multirow[t]{2}{*}{ Reference } \\
\hline & & 9.3 & $\mathrm{H}$ & .20703 & -4 & .13005 & -4 & & & $\begin{array}{l}0.059 \\
0.360 \\
0.418\end{array}$ & $\begin{array}{l}0.12 \\
0.12 \\
0.83\end{array}$ & \\
\hline & $129 m$ & 37 & $D$ & .21683 & -6 & .309625 & -3 & 0 & 0 & 0.1063 & 100 & NUS \\
\hline & 129 & 72 & $M$ & .16045 & -3 & .309625 & -3 & $\begin{array}{l}0.3 \\
0.7 \\
0.99 \\
1.453\end{array}$ & $\begin{array}{r}1 \\
3 \\
16 \\
80\end{array}$ & $\begin{array}{l}0.0268 \\
0.475 \\
0.72 \\
0.211 \\
1.12\end{array}$ & $\begin{array}{l}80 \\
16 \\
31.5 \\
31.5 \\
1\end{array}$ & NDS, NIJS \\
\hline & $131 \mathrm{~m}$ & 30 & $\mathrm{H}$ & .64181 & -5 & .23009 & -2 & $\begin{array}{l}0.215 \\
0.420 \\
0.570 \\
2.457\end{array}$ & $\begin{array}{c}3.0 \\
43 \\
31 \\
3.8\end{array}$ & $\begin{array}{l}0.08 \\
0.100 \\
0.200 \\
0.240 \\
0.335 \\
0.780 \\
0.840 \\
0.920 \\
1.08 \\
1.14 \\
1.22 \\
1.620 \\
1.92 \\
2.0 \\
2.24\end{array}$ & $\begin{array}{r}4 \\
8 \\
8 \\
3 \\
16 \\
80 \\
40 \\
5 \\
5 \\
25 \\
17 \\
3 \\
2 \\
4 \\
15\end{array}$ & NUS \\
\hline & 131 & 24 & $M$ & .48135 & -3 & .23009 & -2 & $\begin{array}{l}1.15 \\
1.36 \\
1.68 \\
2.14\end{array}$ & $\begin{array}{r}10 \\
5 \\
25 \\
60\end{array}$ & $\begin{array}{l}0.145 \\
0.454 \\
0.600 \\
0.920 \\
0.985 \\
1.13\end{array}$ & $\begin{array}{r}80 \\
20 \\
5 \\
5 \\
1 \\
10\end{array}$ & NUS \\
\hline & 132 & 78 & $\mathrm{H}$ & .24685 & -5 & .1220488 & -1 & 0.22 & 100 & $\begin{array}{l}0.052 \\
0.233\end{array}$ & $\begin{array}{l}100 \\
100\end{array}$ & NDS \\
\hline
\end{tabular}




\begin{tabular}{|c|c|c|c|c|c|c|c|c|c|c|c|c|}
\hline \multirow[t]{2}{*}{ Nuclide } & \multirow{2}{*}{$\begin{array}{c}\text { Mass } \\
\text { No. }\end{array}$} & \multicolumn{2}{|c|}{ Half-life } & \multicolumn{2}{|c|}{$\begin{array}{c}\text { Decay Constant } \\
\left(\sec ^{-1}\right)\end{array}$} & \multicolumn{2}{|c|}{$\begin{array}{l}\text { Fission Yield } \\
\text { (Fractional) }\end{array}$} & \multirow{2}{*}{ 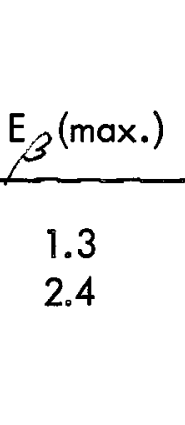 } & \multirow{2}{*}{$\begin{array}{l}\% \\
70 \\
17\end{array}$} & \multirow{2}{*}{$\begin{array}{l}E_{\gamma} \\
0.334 \\
0.432 \\
0.754 \\
0.91\end{array}$} & \multirow{2}{*}{$\begin{array}{c}-20- \\
\% \\
13 \\
70 \\
100 \\
70\end{array}$} & \multirow{2}{*}{$\frac{\text { Reference }}{\text { NDS }}$} \\
\hline & & 52 & $M$ & .22216 & -3 & 1305522 & -1 & & & & & \\
\hline & 133 & 2 & $M$ & .57762 & -2 & .1305522 & -1 & 2.4 & 100 & 0 & 0 & NIJS \\
\hline & 134 & 44 & $M$ & .26256 & -3 & .3781513 & -1 & 1.4 & 100 & & & NIJS \\
\hline & 135 & 1.4 & $M$ & .82518 & -2 & .2250900 & -1 & 3 & 100 & 3 & 100 & NDS* \\
\hline \multirow[t]{4}{*}{531} & 129 & 1.6 & $7 Y$ & $\begin{array}{l}13727 \\
2920\end{array}$ & -14 & .0 & & 0.15 & 100 & 0.040 & 100 & NDS \\
\hline & 130 & 12.6 & $\mathrm{H}$ & .15281 & -4 & .5622 & -4 & $\begin{array}{l}0.60 \\
1.02\end{array}$ & $\begin{array}{l}47 \\
53\end{array}$ & $\begin{array}{l}0.410 \\
0.53 \\
0.66 \\
0.74 \\
1.15\end{array}$ & $\begin{array}{r}24 \\
100 \\
100 \\
70 \\
30\end{array}$ & NDS \\
\hline & 131 & 8.05 & $D$ & .99659 & -6 & .9454 & -4 & $\begin{array}{l}0.250 \\
0.33 \\
0.606 \\
0.81\end{array}$ & $\begin{array}{r}2.8 \\
9.1 \\
87.5 \\
0.7\end{array}$ & $\begin{array}{l}0.284 \\
0.364 \\
0.638 \\
0.724\end{array}$ & $\begin{array}{l}5.5 \\
81 \\
9.1 \\
2.8\end{array}$ & NIJS \\
\hline & 132 & 2.30 & $H$ & .83714 & -4 & .167067 & -2 & $\begin{array}{l}0.80 \\
1.04 \\
1.22 \\
1.49 \\
1.61 \\
2.14 \\
2.92\end{array}$ & $\begin{array}{l}21 \\
15 \\
12 \\
12 \\
21 \\
18 \\
0.03\end{array}$ & $\begin{array}{l}0.24 \\
0.32 \\
0.46 \\
0.48 \\
0.52 \\
0.62 \\
0.647 \\
0.65 \\
0.673 \\
0.72 \\
0.775 \\
0.95 \\
1.14\end{array}$ & $\begin{array}{c}1.3 \\
2.2 \\
19 \\
5.2 \\
9 \\
26 \\
99 \\
6.5 \\
82+ \\
19 \\
4.5\end{array}$ & NUS \\
\hline
\end{tabular}




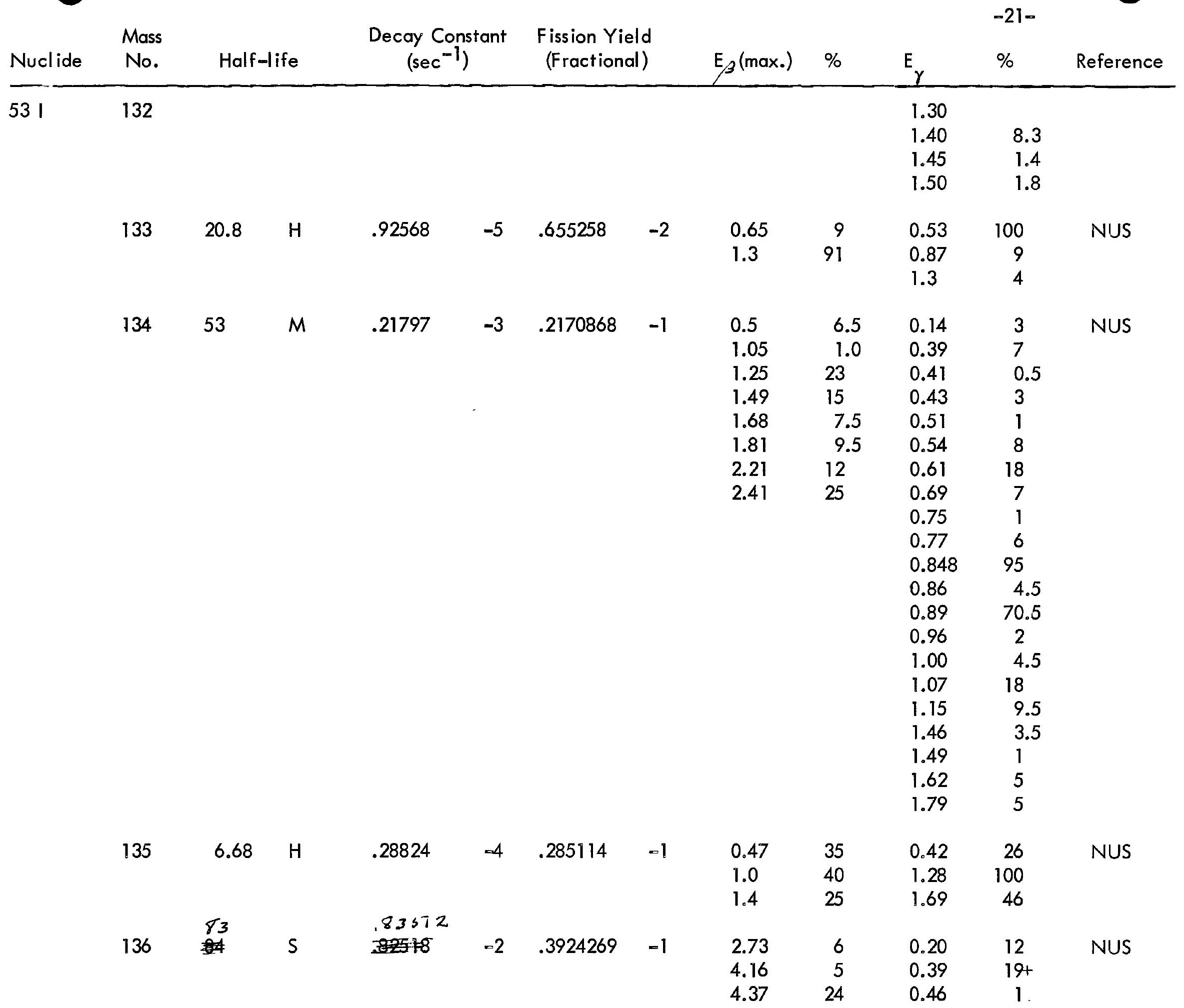




\begin{tabular}{|c|c|c|c|c|c|c|c|c|c|c|c|c|}
\hline \multirow{2}{*}{$\frac{\text { Nuclide }}{53 \text { I }}$} & \multirow{2}{*}{$\begin{array}{c}\begin{array}{c}\text { Mass } \\
\text { No. }\end{array} \\
136\end{array}$} & \multicolumn{2}{|c|}{ Half-life } & \multicolumn{2}{|c|}{$\begin{array}{c}\text { Decay Constant } \\
\left(\sec ^{-1}\right)\end{array}$} & \multicolumn{2}{|c|}{$\begin{array}{l}\text { Fission Yield } \\
\text { (Fractional) }\end{array}$} & $\mathrm{E}_{\beta}\left(\max x_{0}\right)$ & $\%$ & $\mathrm{E}_{\gamma}$ & $\begin{array}{c}-22- \\
\%\end{array}$ & Reference \\
\hline & & & & & & & & 5.60 & 15 & 0.53 & & \\
\hline & & & & & & & & 7.0 & 6 & 0.71 & 3 & \\
\hline & & & & & & & & 3.5 & 44 & 1.00 & 6 & \\
\hline & & & & & & & & & & 1.32 & 95 & \\
\hline & & & & & & & & & & 1.55 & 4 & \\
\hline & & & & & & & & & & 1.72 & 2 & \\
\hline & & & & & & & & & & 1.91 & 5 & \\
\hline & & & & & & & & & & 2.25 & 7 & \\
\hline & & & & & & & & & & 2.40 & 13 & \\
\hline & & & & & & & & & & 2.63 & 11 & \\
\hline & & & & & & & & & & 2.84 & 8 & \\
\hline & & & & & & & & & & 3.2 & 5 & \\
\hline & 137 & 24.4 & $S$ & .28408 & -1 & .2661064 & -1 & 2.75 & 100 & 2.75 & 100 & NDS \\
\hline & 138 & 6.3 & $S$ & .11002 & 0 & .1457583 & -1 & 3.9 & 100 & 3.9 & 100 & NDS* \\
\hline & 139 & 2.0 & $S$ & .34657 & 0 & .784884 & -2 & 3.5 & 100 & 3.5 & 100 & NDS* \\
\hline $54 \times \mathrm{e}$ & $131 \mathrm{~m}$ & 12 & $D$ & .66855 & -6 & 0 & 0 & 0 & 0 & 0.164 & 100 & NUS \\
\hline & $133 m$ & 2.3 & $\mathrm{D}$ & .34881 & -5 & 0 & 0 & 0 & 0 & 0.233 & 100 & NUS \\
\hline & 133 & 5.27 & D & .15223 & -5 & 0 & 0 & 0.268 & 1 & 0.079 & 0.8 & NDS \\
\hline & & & & & & & & 0.347 & 99 & 0.081 & 100 & \\
\hline & $135 m$ & $\begin{array}{l}15.3 \\
-6.6\end{array}$ & $M$ & $\begin{array}{r}.75505 \\
\square\end{array}$ & -3 & .465186 & -2 & 0 & 0 & 0.53 & 100 & NUS \\
\hline & 135 & 9.2 & $H$ & .20928 & -4 & .465186 & -2 & 0.55 & 3 & 0.250 & 97 & NUS \\
\hline & & & & & & & & 0.91 & 97 & 0.36 & $<1$ & \\
\hline & & & & & & & & & & 0.604 & 3 & \\
\hline & 137 & 3.9 & $M$ & .29622 & -2 & .2661064 & -1 & 3.5 & 100 & 0 & 0 & NUS \\
\hline & 138 & 17 & $M$ & .67956 & -3 & .2751100 & -1 & 2.4 & 100 & 0.42 & 100 & NUS \\
\hline & 139 & 41 & $S$ & .16906 & -1 & .2751100 & -1 & 4.6 & 100 & & & NUS \\
\hline
\end{tabular}




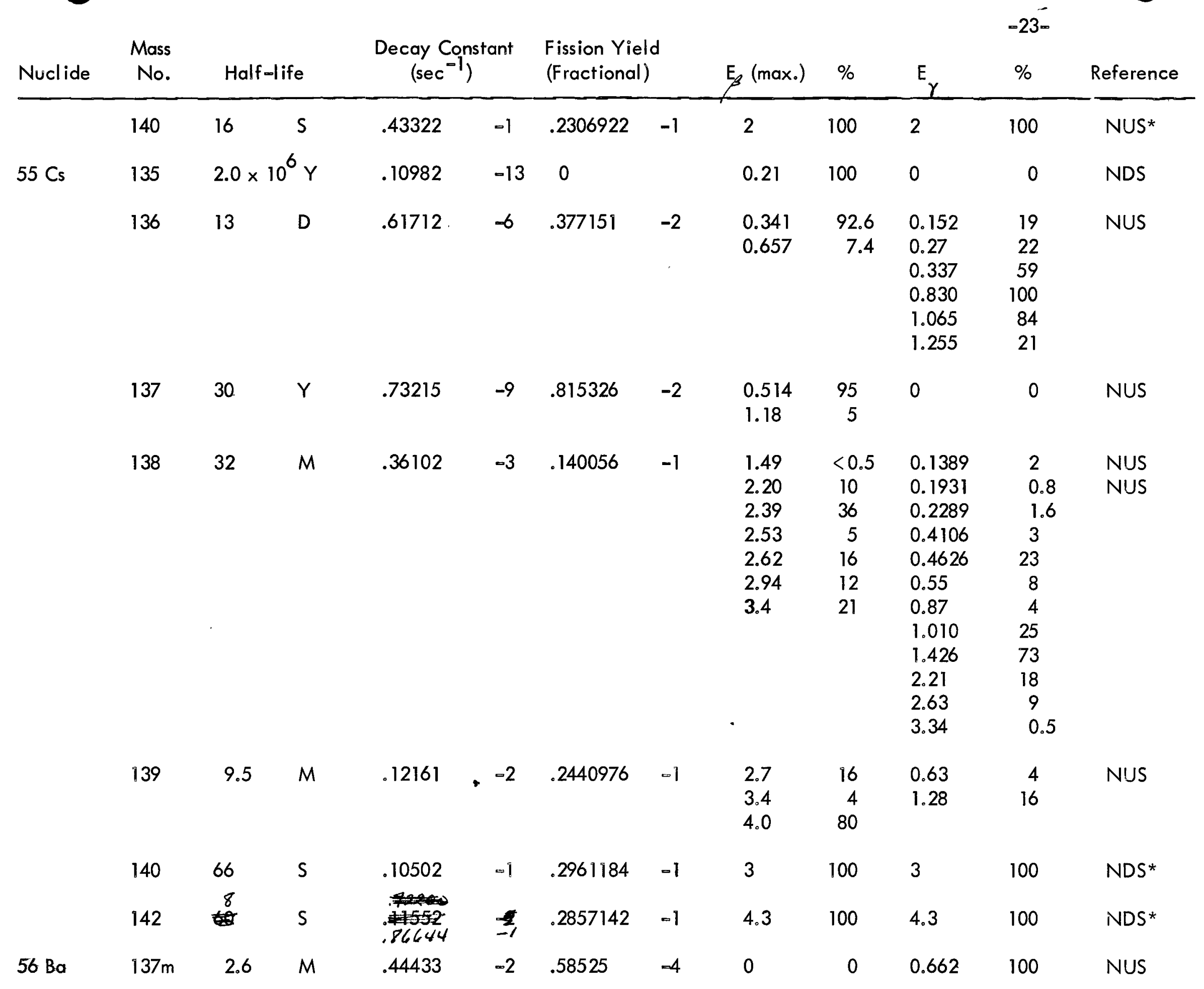




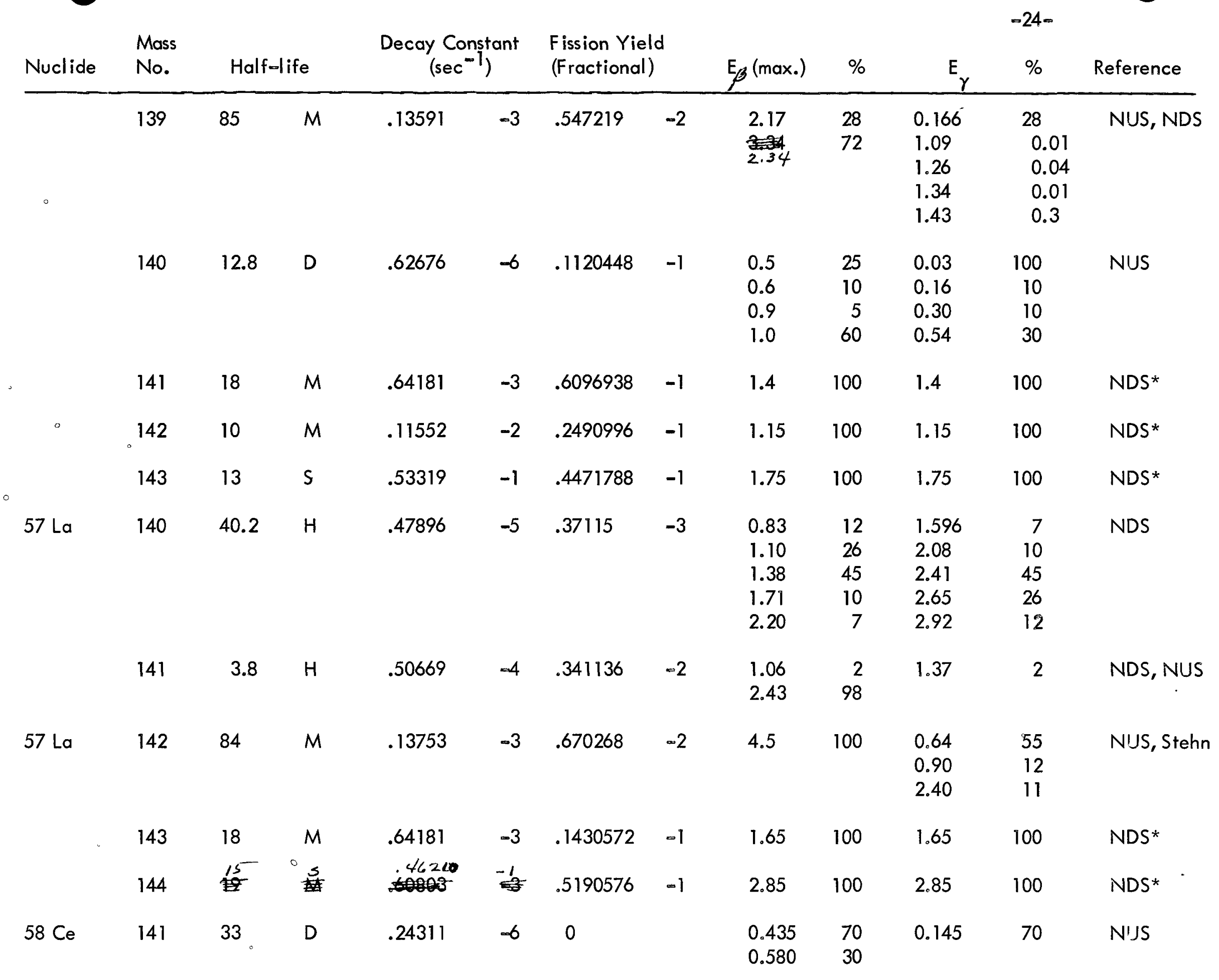




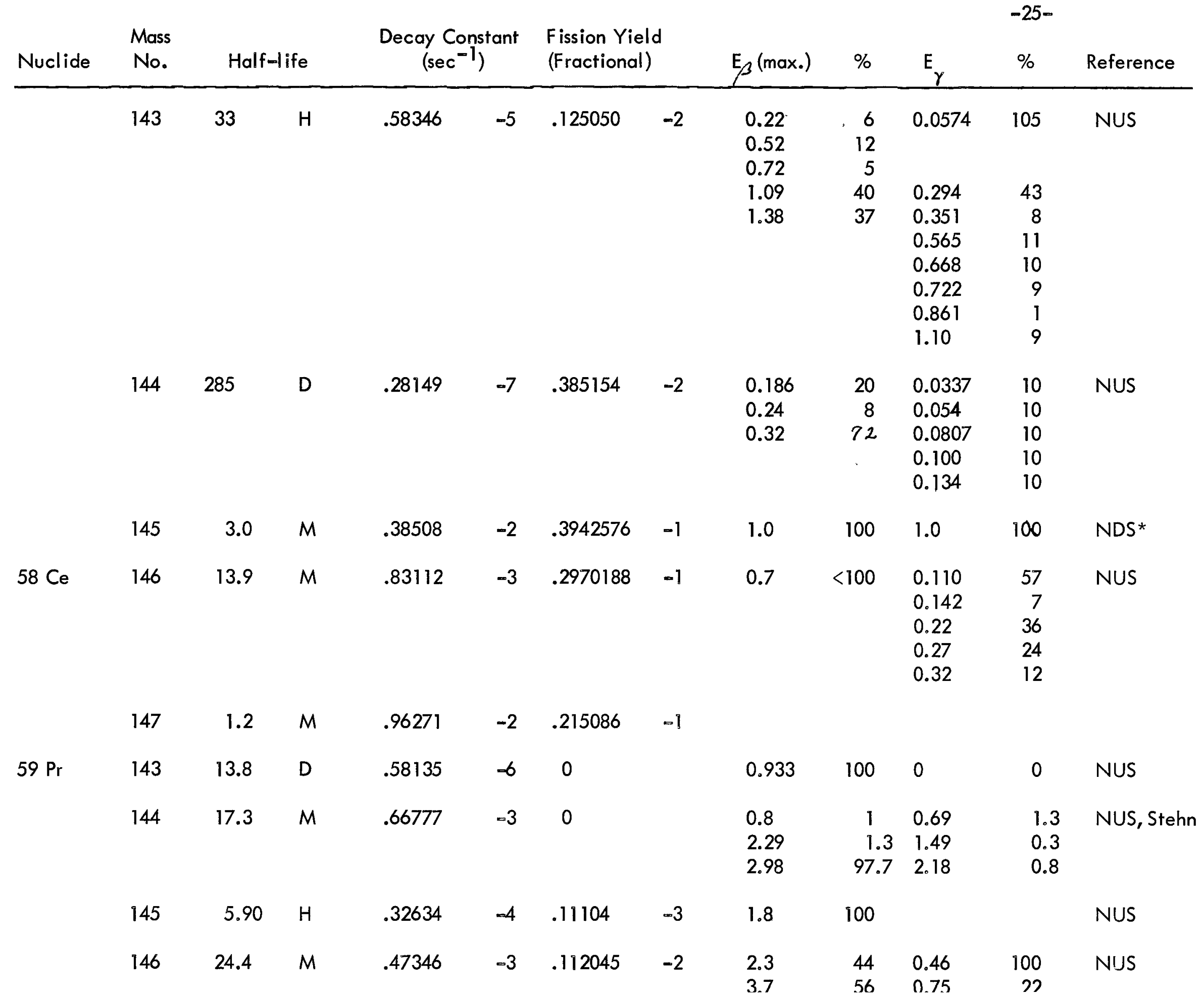




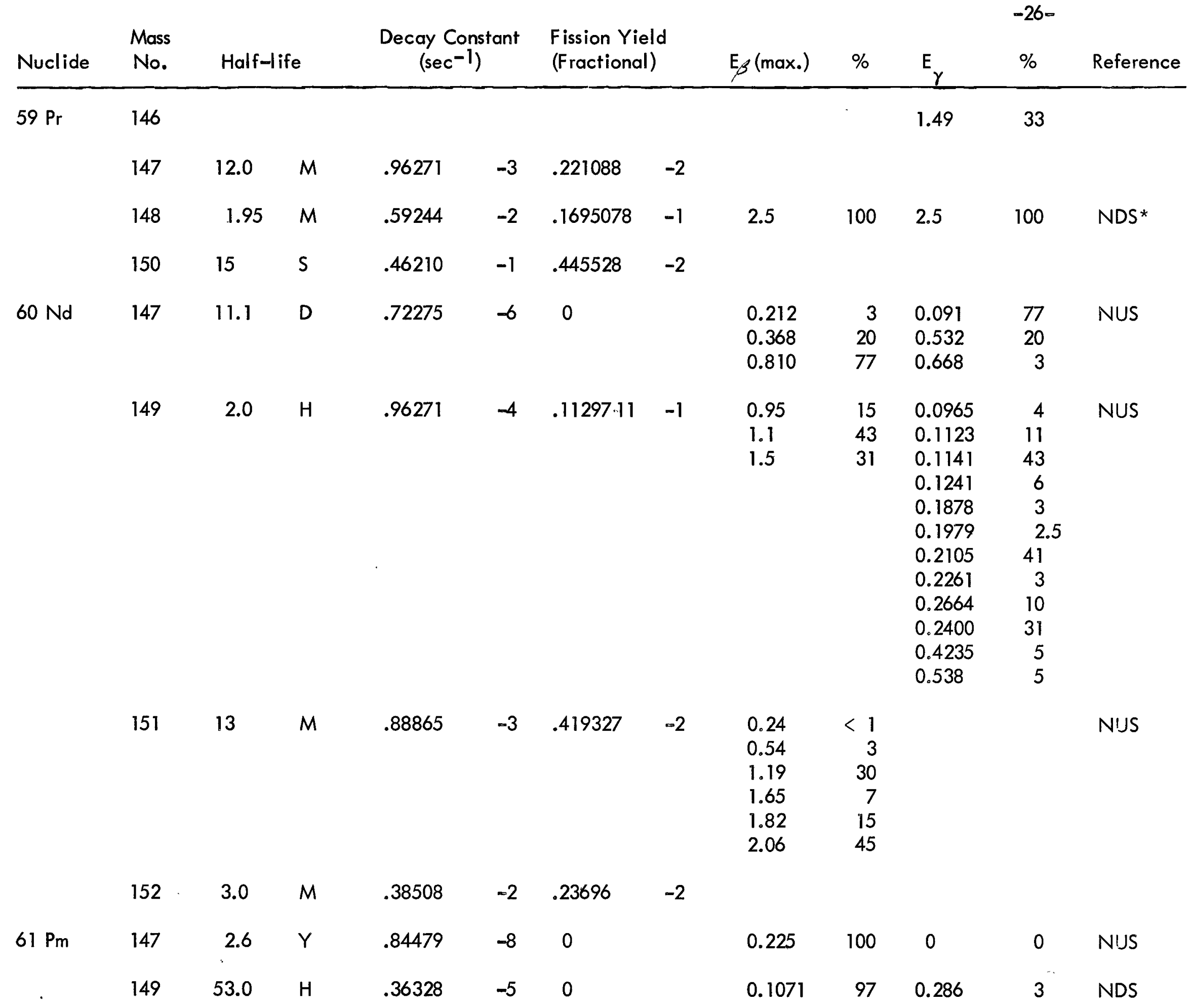




\begin{tabular}{|c|c|c|c|c|c|c|c|c|c|c|c|c|}
\hline \multirow{2}{*}{$\frac{\text { Nuclide }}{61 \mathrm{Pm}}$} & \multirow{2}{*}{$\begin{array}{l}\text { Mass } \\
\text { No. } \\
149\end{array}$} & \multicolumn{2}{|c|}{ Half-life } & \multicolumn{2}{|c|}{$\begin{array}{c}\text { Decay Constant } \\
\left(\sec ^{-1}\right)\end{array}$} & \multicolumn{2}{|c|}{$\begin{array}{c}\text { Fission Yield } \\
\text { (Fractional }\end{array}$} & $\left.\mathrm{E}_{\beta}(\max )_{0}\right)$ & $\%$ & $E_{\gamma}$ & $\begin{array}{l}-27- \\
\%\end{array}$ & Reference \\
\hline & & & & & & & & 0.784 & 3 & & & \\
\hline & 150 & 2.7 & $\mathrm{H}$ & .71312 & -4 & .21208 & -3 & $\begin{array}{l}2.0 \\
3.0\end{array}$ & $\begin{array}{l}80 \\
20\end{array}$ & $\begin{array}{l}0.34 \\
0.40 \\
0.82 \\
1.17\end{array}$ & $\begin{array}{r}20 \\
20 \\
\cdot 80 \\
80\end{array}$ & NDS \\
\hline & 151 & 28.4 & $\mathrm{H}$ & .67796 & -5 & .23209 & -3 & 1.1 & 100 & 0.715 & 100 & NIJS \\
\hline & 152 & 6.0 & $M$ & .19254 & -2 & .38315 & -3 & 2.2 & 100 & 2.8 & 100 & NDS* \\
\hline & 153 & $\begin{array}{l}5.5 \\
=\end{array}$ & $M$ & $.21005^{-}$ & -2 & .165206 & -2 & & & & & \\
\hline & 154 & $\begin{array}{l}2.5 \\
3\end{array}$ & $M$ & $\begin{array}{r}46210 \\
-10\end{array}$ & -2 & .69167 & -3 & 1.25 & 100 & 1.25 & 100 & NDS* \\
\hline $62 \mathrm{Sm}$ & 151 & 80 & $Y$ & .27456 & -9 & 0 & & $\begin{array}{l}0.053 \\
0.075\end{array}$ & 1 & 0.0216 & 1 & NUS \\
\hline & 153 & 47 & $\mathrm{H}$ & .40966 & -5 & .6032 & -4 & $\begin{array}{l}0.64 \\
0.698 \\
0.803\end{array}$ & $\begin{array}{l}38 \\
40 \\
22\end{array}$ & $\begin{array}{l}0.0697 \\
0.1032 \\
0.538\end{array}$ & $\begin{array}{l}38 \\
78 \\
10\end{array}$ & NUS \\
\hline & 155 & 24 & $M$ & .48135 & -3 & .32353 & -3 & $\begin{array}{l}1.50 \\
1.65\end{array}$ & $\begin{array}{r}7 \\
93\end{array}$ & $\begin{array}{l}0.105 \\
0.246\end{array}$ & $\begin{array}{r}93 \\
7\end{array}$ & NUS \\
\hline & 156 & 10 & $H$ & .19254 & -4 & .13358 & -3 & 0.9 & 100 & 0 & 0 & NUS \\
\hline $63 \mathrm{Eu}$ & 155 & 1.7 & $Y$ & .12920 & -7 & .2490 & -5 & $\begin{array}{l}0.15 \\
0.16 \\
0.19 \\
0.25\end{array}$ & $\begin{array}{r}30 \\
40 \\
<10 \\
20\end{array}$ & $\begin{array}{c}0.060 \\
0.019 \\
<0.45 \\
0.105 \\
0.27 \\
0.87\end{array}$ & $\begin{array}{l}<10 \\
30 \\
30 \\
40 \\
40\end{array}$ & NUS \\
\hline & 156 & 15.4 & $D$ & .52095 & -6 & .7380 & -5 & $\begin{array}{l}0.5 \\
2.4\end{array}$ & $\begin{array}{l}60 \\
40\end{array}$ & 2.0 & 60 & NDS \\
\hline
\end{tabular}




\begin{tabular}{|c|c|c|c|c|c|c|c|c|c|c|c|c|}
\hline Nuclide & \multirow{2}{*}{$\begin{array}{l}\text { Mass } \\
\text { No. }\end{array}$} & \multicolumn{2}{|c|}{ Half-life } & \multicolumn{2}{|c|}{$\begin{array}{l}\text { Decay Constant } \\
\left(\sec ^{-1}\right)\end{array}$} & \multicolumn{2}{|c|}{$\begin{array}{l}\text { Fission Yield } \\
\text { (Fractional) }\end{array}$} & $E_{\beta}(\max )$. & $\%$ & $E_{\gamma}$ & $\begin{array}{c}-28- \\
\%\end{array}$ & Reference \\
\hline & & 15.0 & $\mathrm{H}$ & .12836 & -4 & .7719 & -4 . & $\begin{array}{l}1.0 \\
1.7\end{array}$ & $\begin{array}{l}75 \\
25\end{array}$ & $\begin{array}{l}0.2 \\
0.6\end{array}$ & $\begin{array}{l}100 \\
100\end{array}$ & NIJS \\
\hline & 160 & 70 & $S$ & .99021 & -2 & .2800 & -5 & & & & & \\
\hline $64 \mathrm{Gd}$ & 159 & 18.0 & $\mathrm{H}$ & 10697 & -4 & .1047 & -4 & $\begin{array}{l}0.59 \\
0.89 \\
0.948\end{array}$ & $\begin{array}{r}20 \\
6 \\
74\end{array}$ & $\begin{array}{l}0.058 \\
0.079 \\
0.225 \\
0.362\end{array}$ & $\begin{array}{c}6.6 \\
0.6 \\
0.6 \\
19\end{array}$ & N'JS \\
\hline
\end{tabular}




\begin{tabular}{|c|c|c|c|c|c|c|c|c|c|c|c|c|}
\hline \multirow{2}{*}{$\frac{\text { Nuclide }}{65 \mathrm{~Tb}}$} & \multirow{2}{*}{$\begin{array}{c}\begin{array}{c}\text { Mass } \\
\text { No. }\end{array} \\
160\end{array}$} & \multicolumn{2}{|c|}{ Half-life } & \multicolumn{2}{|c|}{$\begin{array}{c}\text { Decay Constant } \\
\left(\sec ^{-1}\right)\end{array}$} & \multicolumn{2}{|c|}{$\begin{array}{c}\text { Fission Yield } \\
\text { (Fractional) }\end{array}$} & $\mathrm{E}_{\beta}$ (max.) & $\%$ & \multicolumn{2}{|c|}{$\begin{array}{l}-29- \\
\%\end{array}$} & Reference \\
\hline & & & & & & & & & & $\begin{array}{l}0.964 \\
1.18\end{array}$ & $\begin{array}{l}35 \\
16\end{array}$ & \\
\hline & 161 & 6.8 & D & .11798 & -5 & .3000 & -7 & $\begin{array}{l}0.41 \\
0.46 \\
0.49 \\
0.54\end{array}$ & $\begin{array}{l}17 \\
11 \\
12 \\
60\end{array}$ & $\begin{array}{l}0.0257 \\
0.0487 \\
0.0571 \\
0.0745\end{array}$ & & NDS \\
\hline
\end{tabular}

